\title{
SUR L'UNICITÉ DU CÔNE CONVEXE DIVISIBLE RELATIF AU NOYAU DE CONVOLUTION DE HUNT DÉFINI PAR L'OPÉRATEUR DIFFÉRENTIEL
}

\author{
MASAYUKI ITÔ
}

\section{§1. Introduction}

Dans toute la suite $\boldsymbol{R}^{n}$ désignera l'espace euclidien à dimension $n(\geqq 1)$. Pour un point $x=\left(x_{1}, x_{2}, \cdots, x_{n}\right)$ de $\boldsymbol{R}^{n}$, on note $|x|=\left(\sum_{i=1}^{n} x_{i}{ }^{2}\right)^{1 / 2}$ et la coordonnée sphérique dans $\boldsymbol{R}^{n}$ désignera $(r, \sigma)$.

Rappelons qu'un noyau de convolution $N$ sur $\boldsymbol{R}^{n}$ est une mesure (de Radon) positive dans $\boldsymbol{R}^{n}$ dans la théorie du potentiel. Pour une mesure réelle $\mu$ dans $\boldsymbol{R}^{n}, N * \mu$ s'appelle le $N$-potentiel de $\mu$ dès que cette convolution est définie au sens des mesures. On connaît bien que, dans la théorie du potentiel, les noyaux de convolution de Hunt possédent les propriétés définitives.

Dans l'article [6], on a introduit la notion du cône convexe divisible (du cône convexe de Riesz) relatif au noyau de convolution de Hunt donné et on a montré, dans l'article [7], que pour un opérateur différentiel elliptique et auto-adjoint $L$ d'ordre $\leqq 2$ sur $\boldsymbol{R}^{n}$ à coéfficients constants tel qu'il existe le noyau de convolution $G_{L}$ sur $\boldsymbol{R}^{n}$ s'annulant à l'infini vérifiant $L G_{L}=-\varepsilon$ (au sens des distributions), où $\varepsilon$ est la mesure d'unité à l'origine, un cône convexe divisible relatif au noyau $G_{L}$ formé par 0 et de noyaux de convolution de Dirichlet sur $\boldsymbol{R}^{n}$ est uniquement déterminé et que cela est égal à

$$
\left\{c \varepsilon+\int G_{L, p} d \nu(p) ; c \in \boldsymbol{R}^{+}, \nu \in M^{+}\left(\boldsymbol{R}^{+}\right) \text {avec } \int_{1}^{\infty} \frac{1}{p} d \nu(p)<+\infty\right\},
$$

où $\boldsymbol{R}^{+}=\left\{t \in \boldsymbol{R}^{1} ; t \geqq 0\right\}, M^{+}\left(\boldsymbol{R}^{+}\right)$est l'ensemble formé par toutes les mesures positives sur $\boldsymbol{R}^{+}$et où $\left(G_{L, p}\right)_{p \geqq 0}$ est la résolvante associée au noyau $G_{L}$.

Le but de cette note est une généralisation de ce résultat. Soit $L$ 
un opérateur différentiel elliptique d'ordre $\leqq 2$ sur $\boldsymbol{R}^{n}$ à coéfficients constants tel qu'il existe le noyau de convolution de Hunt $G_{L}$ sur $\boldsymbol{R}^{n}$ à $L G_{L}=-\varepsilon$. Alors un cône convexe divisible relatif au noyau $G_{L}$ est toujours unique et cela est de la même forme que ci-dessus.

Rappelons finalement la définition d'un cône convexe divisible $C_{R}\left(N_{0}\right)$ relatif au noyau de convolution de Hunt donné $N_{0}$ sur $R^{n}: C_{R}\left(N_{0}\right)$ est un cône convexe vaguement fermé de vertex 0 constitué par 0 et de noyaux de convolution de Hunt sur $\boldsymbol{R}^{n}$ et vérifiant

(a) $N_{0} \in C_{R}\left(N_{0}\right)$

(b) À tout l'élément $N \neq 0$ de $C_{R}\left(N_{0}\right)$, on peut associer un élément $N^{\prime} \neq 0$ de $C_{R}\left(N_{0}\right)$ tel que $N * N^{\prime}=N_{0}$.

On remarque que, dans ce cas, $N^{\prime}$ est uniquement déterminé.

\section{§2. Préliminaires}

D'après C. S. Herz [3], on dit qu'une distribution $u$ dans $\boldsymbol{R}^{n}$ est conditionnellement positive si $u \geqq 0$ au sens des distributions en dehors de l'origine. Rappelons que toute la distribution est localement d'ordre fini et posons, pour un entier $k \geqq 1$,

$\mathcal{O}^{k}\left(\boldsymbol{R}^{n}\right)=\left\{f \in C_{K}^{\infty}\left(\boldsymbol{R}^{n}\right) ; D^{\alpha} f(0)=0\right.$ pour tout le multi-indice $\alpha$ à $\left.|\alpha|<k\right\}$, où $C_{K}^{\infty}\left(\boldsymbol{R}^{n}\right)$ est l'espace de $(L F)$ usuel des fonctions infiniment dérivables dans $R^{n}$ à valeurs complexes et à support compact et où, pour $\alpha$ $=\left(\alpha_{1}, \cdots, \alpha_{n}\right), D^{\alpha}=\frac{\partial^{|\alpha|}}{\partial x_{1}^{\alpha_{1}} \cdots \partial x_{n}^{\alpha_{n}}}$ et $|\alpha|=\sum_{i=1}^{n} \alpha_{i}$. Alors on obtient qu'une distribution $u$ est conditionnellement positive si et seulement s'il existe un entier $k \geqq 1$ tel que $u$ soit $k$-conditionnellement positive; c'est-à-dire, quelle que soit $\varphi$ de $\mathcal{O}^{k}\left(\boldsymbol{R}^{n}\right), u\left(|\varphi|^{2}\right) \geqq 0$. Pour un entier $k \geqq 1$, on note $C P^{k}=C P^{k}\left(\boldsymbol{R}^{n}\right)$ l'ensemble constitué par toutes les distributions $k$-conditionnelement positives dans $\boldsymbol{R}^{n}$, et alors, d'après $\mathcal{O}^{k}\left(\boldsymbol{R}^{n}\right) \supset \mathcal{O}^{k+1}\left(\boldsymbol{R}^{n}\right), C P^{k}$ $\subset C P^{k+1}$. On note ensuite $C P=\bigcup_{k=1}^{\infty} C P^{k}$. Soit $u \in C P^{k}$ et supposons que $u$ est à croissance lente. On a alors, pour toute $\varphi$ de $\mathcal{O}^{k}\left(R^{n}\right)$,

$$
\hat{u} * \hat{\varphi} * \tilde{\varphi}(0)=u\left(|\varphi|^{2}\right) \geqq 0,
$$

où $\wedge$ représente la transformation de Fourier et $\tilde{\hat{\varphi}}(x)=\overline{\hat{\varphi}(-x)}$. Posons, pour un entier $k \geqq 1$,

$$
\hat{\mathcal{O}}^{k}\left(\boldsymbol{R}^{n}\right)=\left\{\varphi \in C_{K}^{\infty}\left(\boldsymbol{R}^{n}\right) ; \int x^{\alpha} \varphi(x) d x=0 \text { pour tout le multi-indice } \alpha \text { à }|\alpha|<k\right\},
$$


où, pour $\alpha=\left(\alpha_{1}, \cdots, \alpha_{n}\right), x^{\alpha}=x_{1}^{\alpha_{1}} \cdots x_{n}^{\alpha_{n}}$. On dit, d'après [3], qu'une distribution $v$ dans $\boldsymbol{R}^{n}$ est $k$-conditionnellement de type positif si, quelle que soit $\varphi$ de $\hat{\mathscr{O}}^{k}\left(\boldsymbol{R}^{n}\right), v * \varphi * \tilde{\varphi}(0) \geqq 0$. On note $C T P^{k}=C T P^{k}\left(\boldsymbol{R}^{n}\right)$ la totalité des distributions $k$-conditionnellement de type positif dans $\boldsymbol{R}^{n}$, et alors $C T P^{k} \subset C T P^{k+1}$. On pose $C T P=\bigcup_{k=1}^{\infty} C T P^{k}$ et toute la distribution appartenant à $C T P$ est simplement dite conditionnellement de type positif. On obtient facilement la proposition suivante (cf. [3]).

Proposition 1. Soit $k$ un entier $\geqq 1$. Alors les deux énoncés suivants sont équivalents.

(1) $v \in C T P^{k}$.

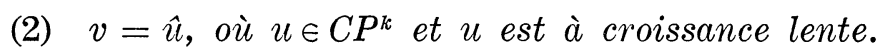

Donc on obtient facilement que $v \in C T P$ si et seulement si $v=\hat{u}$, où $u$ est conditionnellement positive (c'est-à-dire, $u \in C P$ ) et à croissance lente. C. S. Herz a montré que toute la fonction de $\widehat{\mathscr{O}}^{k}$ est de la forme explicite suivante (cf. [3]).

Proposition 2. Pour un entier $k \geqq 1$, $\mathcal{O}^{k}$ est un idéal de convolution de $C_{K}^{\infty}\left(\boldsymbol{R}^{n}\right)$ et on a

$$
\hat{\mathscr{O}}^{k}=\hat{\mathscr{O}}^{k}\left(\boldsymbol{R}^{n}\right)=\left\{\sum_{|| \alpha \mid=k} D^{\alpha} g_{\alpha} ; g_{\alpha} \in C_{K}^{\infty}\left(\boldsymbol{R}^{n}\right)\right\} .
$$

Soit $u$ une distribution dans $\boldsymbol{R}^{n}$. On dit que $u$ est un laplacien généralisé sur $\boldsymbol{R}^{n}$ si l'on a, quelle que soit $\varphi$ de $C_{K}^{\infty}\left(\boldsymbol{R}^{n}\right), u(\varphi) \leqq 0$ dès que $\varphi$ est à valeurs réelles et $\varphi(0)=\max _{v \in R^{n}} \varphi(x)$, et alors $u \in C P^{1}$.

Proposition 3 (cf. par exemple, [3]). Soit u une distribution dans $\boldsymbol{R}^{n}$. Alors les trois énoncés suivants sont équivalents.

(1) $u$ est un laplacien généralisé sur $\boldsymbol{R}^{n}$.

(2) - $\hat{u}$ est égale à une fonction définie-négative $\psi$ dans $\boldsymbol{R}^{n}$.

(3) Il existe un semi-groupe vaguement continu $\left(\alpha_{t}\right)_{t \geqq 0}$ de mesures positives dans $\boldsymbol{R}^{n} \grave{a} \int d \alpha_{t} \leqq 1(\forall t \geqq 0)$ tel que $\frac{1}{t}\left(\alpha_{t}-\varepsilon\right)$ converge vers $u$ dans $\boldsymbol{R}^{n}$ au sens des distributions dans $\boldsymbol{R}^{n}$ lorsque $t \rightarrow 0$.

Une fonction définie-négative $\psi$ dans $\boldsymbol{R}^{n}$ est, par définition, une fonction complexe et continue dans $\boldsymbol{R}^{n}$ qui vérifie les deux conditions suivantes: 
(a) $\psi(0) \geqq 0$ et $\psi(-x)=\overline{\psi(x)}$ pour tout $x$ de $\boldsymbol{R}^{n}$.

(b) Quels que soient $m$ un entier $\geqq 1$, $\left(x^{i}\right)_{i=1}^{m}$ et $\left(c_{i}\right)_{i=1}^{m}$, une famille de points de $\boldsymbol{R}^{n}$ et une famille de nombres complexes à $\sum_{i=1}^{m} c_{i}=0$,

$$
\sum_{i=1}^{m} \sum_{j=1}^{m} \psi\left(x^{i}-x^{j}\right) c_{i} \bar{c}_{j} \leqq 0
$$

D'après le théorème de Levy-Khinchine, pour une fonction définienégative $\psi$ dans $\boldsymbol{R}^{n}$, il existe une famille d'une constante $c \geqq 0$, d'une forme linéaire $L(x)$ sur $\boldsymbol{R}^{n}$, d'une forme quadratique $Q(x) \geqq 0$ sur $\boldsymbol{R}^{n}$ et d'une mesure positive $\alpha$ en dehors de l'origine à $\int \frac{|x|^{2}}{1+|x|^{2}} d \alpha(x)<+\infty$, et une seule telle que l'on ait

$$
\begin{aligned}
\psi(x)= & c+L(x) \sqrt{-1}+Q(x) \\
& +\int\left(1-\exp (2 \pi \sqrt{-1} x \cdot y)-\frac{2 \pi x \cdot y \sqrt{-1}}{1+x^{2}}\right) d \alpha(y) \text { sur } R^{n},
\end{aligned}
$$

où $x \cdot y$ est le produit scalaire entre $x$ et $y$ sur $\boldsymbol{R}^{n}$.

Une famille $\left(\alpha_{t}\right)_{t \geqq 0}$ de mesures positives dans $\boldsymbol{R}^{n}$ s'appelle un semigroupe vaguement continu si l'on a, pour tous $t \geqq 0, s \geqq 0, \alpha_{t} * \alpha_{s}=\alpha_{t+s}$, $\alpha_{0}=\varepsilon$ et si l'application $t \in \boldsymbol{R}^{+} \rightarrow \alpha_{t}$ est vaguement continue.

Dans la proposition $3,\left(\alpha_{t}\right)_{t \geqq 0}$ est uniquement déterminé et on a $\hat{\alpha}_{t}$ $=\exp (-t \psi) \operatorname{sur} \boldsymbol{R}^{n}$.

Un noyau de convolution de Hunt $N$ sur $\boldsymbol{R}^{n}$ est, par définition, de la forme $N=\int_{0}^{\infty} \alpha_{t} d t$, où $\left(\alpha_{t}\right)_{t \geqq 0}$ est un semi-groupe vaguement continu de mesures positives dans $\boldsymbol{R}^{n}$. Dans ce cas, $\left(\alpha_{t}\right)_{t \geqq 0}$ est unique et s'appelle le semi-groupe associé au noyau $N$.

Proposition 4 (cf. [1] et [4]). Soit $N=\int_{0}^{\infty} \alpha_{t} d t$ un noyau de convolution de Hunt sur $\boldsymbol{R}^{n}$. Alors les énoncés suivants sont équivalents:

(1) Il existe un nombre $t_{0}>0$ tel que $\int d \alpha_{t_{0}} \leqq 1$.

(2) Pour tout $t \geqq 0, \int d \alpha_{t} \leqq 1$.

(3) Il existe un laplacien généralisé $u$ dans $\boldsymbol{R}^{n}$ tel que $u * N=-\varepsilon$ dans $\boldsymbol{R}^{n}$.

(4) $N$ est borné(1).

(1) Cela signifie que, quelle que soit $f$ une fonction finie et continue dans $\boldsymbol{R}^{n}$ à support compact, $N * f$ est bornée sur $\boldsymbol{R}^{n}$. 
(5) $N$ est de type positif au sens faible f(2) $^{\text {. }}$

Dans ce cas, $u$ est uniquement déterminé et s'appelle le laplacien généralisé relatif au noyau $N$.

Soient $N$ un noyau de convolution sur $\boldsymbol{R}^{n}$ et $\left(N_{p}\right)_{p \geqq 0}$ une famille de noyaux de convolution sur $\boldsymbol{R}^{n}$. On dit que $\left(N_{p}\right)_{p \geqq 0}$ est la résolvante associée au noyau $N$ si l'on a, pour tous $p \geqq 0, q>0, N_{p}-N_{q}$ $=(q-p) N_{p} * N_{q}$ et $\lim _{p \rightarrow 0} N_{p}=N_{0}=N$ (vaguement). Il est naturel que $\left(N_{p}\right)_{p \geqq 0}$ est unique. Si $N$ est un noyau de convolution sur $R^{n}$ de la forme $N$ $=\int_{0}^{\infty} \alpha_{t} d t$, alors, en posant $N_{p}=\int_{0}^{\infty} \alpha_{t} \exp (-p t) d t\left({ }^{\forall} p \geqq 0\right),\left(N_{p}\right)_{p \geqq 0}$ est la résolvante associée au noyau $N$. Cela résulte facilement du fait que lorsque l'on pose

$$
\kappa=\left\{\begin{array}{ll}
d t & \operatorname{sur} \boldsymbol{R}^{+} \\
0 & \text { dans } \boldsymbol{R}^{1}-\boldsymbol{R}^{+}
\end{array} \text {et } \kappa_{p}=\left\{\begin{array}{ll}
\exp (-p t) d t & \operatorname{sur} \boldsymbol{R}^{+} \\
0 & \operatorname{dans} \boldsymbol{R}^{1}-\boldsymbol{R}^{+}
\end{array} \quad\left({ }^{\forall} p>0\right),\right.\right.
$$

$\left(\kappa_{p}\right)_{p \geqq 0}$ est la résolvante associée au noyau $\kappa$. Le théorème de Bernstein (cf. par exemple, [8]) affirme la proposition suivante:

Proposition 5. Soit $N$ un noyau de convolution sur $\boldsymbol{R}^{1}$. Alors les deux énoncés suivants sont équivalents:

(1) Pour tout l'entier $m \geqq 0,(-1)^{m} \frac{d^{m}}{d t^{m}} N$ est de $C P\left(\boldsymbol{R}^{1}\right)$ et $N=0$ dans $\boldsymbol{R}-\boldsymbol{R}^{+}$.

(2) $N$ est de la forme $N=c \varepsilon+\int \kappa_{p} d \nu(p)$, où $c \in \boldsymbol{R}^{+}$et $\nu \in M^{+}\left(\boldsymbol{R}^{+}\right)$ à $\int_{1}^{\infty} \frac{1}{p} d \nu(p)<+\infty$.

On remarque ici que pour une mesure positive $\nu$ sur $\boldsymbol{R}^{+}, \int \kappa_{p} d \nu(p)$ définit un noyau de convolution sur $R^{1}$ si et seulement si $\int_{1}^{\infty} \frac{1}{p} d \nu(p)<+\infty$, car

$$
\int_{0}^{1} \int \exp (-p t) d \nu(p) d t=\int \frac{1}{p}(1-\exp (-p)) d \nu(p)
$$

(2) Cela signifie que, quelle que soit $f$ une fonction finie et continue dans $\boldsymbol{R}^{n}$ à support compact, $N * f * \breve{f}(0) \geqq 0$, où $\breve{f}(x)=f(-x)$. Dans ce cas, $N$ n'est pas toujours symétrique par rapport à l'origine. 
Soient $\tilde{N}, N$ et $\left(N_{\tilde{p}}\right)_{\tilde{p} \in \underset{m \geqq 1}{\cup}(\boldsymbol{R}+)^{m}}$ deux noyaux de convolution sur $\boldsymbol{R}^{n}$ et une famille de noyaux de convolution sur $\boldsymbol{R}^{n}$. On dit que $\left(N_{\tilde{p}}\right)_{\tilde{p} \in \underset{m \geqq 1}{\cup}(\boldsymbol{R}+)^{m}}$ est la classe divisible associée au noyau $N$ relativement au noyau $\tilde{N}$ si $\left(N_{(p)}\right)_{p \in R^{+}}$est la résolvante associée au noyau $N$ et si, pour tout $\tilde{p}$ $=\left(p_{1}, p_{2}, \cdots, p_{m}\right)$ de $\bigcup_{m \geqq 1}\left(\boldsymbol{R}^{+}\right)^{m}, N_{\tilde{p}} * N_{\left(p_{1}, p_{2}, \cdots, p_{m}, 0\right)}=\tilde{N}$ et $\left(N_{\left(p_{1}, p_{2}, \cdots, p_{m}, p\right)}\right)_{p \in \boldsymbol{R}^{+}}$ est aussi la résolvante associée au noyau $N_{\left(p_{1}, \ldots, p_{m}, 0\right)}$.

Proposition 6. Soient $\tilde{N}$ et $N$ deux noyaux de convolution sur $\boldsymbol{R}^{n}$ et supposons $\tilde{N} \neq 0$. S'il existe la classe divisible $\left(N_{\tilde{p}}\right)_{\tilde{p} \in \underset{m \geqq 1}{\cup}(\boldsymbol{R}+)^{m}}$ associée au noyau $N$ relativement au noyau $\tilde{N}$, alors elle est uniquement déterminée et pour tout $\tilde{p}$ de $\bigcup_{m \geqq 1}\left(\boldsymbol{R}^{+}\right)^{m}, N_{\tilde{p}}$ est un noyau de convolution de Hunt sur $\boldsymbol{R}^{n}$.

- Pour tout $\tilde{p}=\left(p_{1}, p_{2}, \cdots, p_{m}\right)$ de $\bigcup_{m \geqq 1}\left(\boldsymbol{R}^{+}\right)^{m}$, on a $N_{\tilde{p}} * N_{\left(p_{1}, \cdots, p_{m}, 0\right)}=\tilde{N}$, et par suite, d'après $\tilde{N} \neq 0, N_{\tilde{p}} \neq 0$. Donc cette proposition résulte immédiatement de la proposition suivante connue et du principe d'unicité pour les noyaux de convolution de $\mathrm{Hunt}^{(3)}$.

Proposition 7 (cf. [5]). Soit $N$ un noyau de convolution sur $\boldsymbol{R}^{n}$. Alors pour que $N$ soit un noyau de convolution de Hunt sur $\boldsymbol{R}^{n}$, il faut et il suffit que $N \neq 0$ et il existe la résolvante associée au noyau $N$.

\section{§3. Quelques lemmes}

Commençons d'abord avec la définition du principe relatif de domination. Soient $N_{1}$ et $N_{2}$ deux noyaux de convolution sur $\boldsymbol{R}^{n}$. On dit que $N_{1}$ satisfait au principe de domination relatif au noyau $N_{2}$ si, quelles que soient $f$ et $g$ de $C_{K}^{+}, N_{1} * f(x) \leqq N_{2} * g(x)$ sur $R^{n}$ dès que $N_{1} * f(x)$ $\leqq N_{2} * g(x)$ sur le support $\operatorname{supp}(f)$ de $f$. Dans cette note, on note toujours $C_{K}=C_{K}\left(\boldsymbol{R}^{n}\right)$ et $C_{K}^{+}=C_{K}^{+}\left(\boldsymbol{R}^{n}\right)$ l'espace de $(L F)$ usuel des fonctions finies et continues dans $\boldsymbol{R}^{n}$ à support compact et son sous-ensemble des fonctions non-négatives, respectivement.

LEMme 1. Soient $N_{1}$ et $N_{2}$ deux noyaux de convolution $\neq 0$ sur $\boldsymbol{R}^{n}$. Si $N_{1}$ satisfait au principe de domination relatif au noyau $N_{2}$, alors on $a \operatorname{supp}\left(N_{1}\right)+\operatorname{supp}\left(N_{2}\right) \subset \operatorname{supp}\left(N_{2}\right)$.

(3) Un noyau de convolution $N$ sur $\boldsymbol{R}^{n}$ satisfait au principe d'unicité si, quelle que soit $\mu$ une mesure réelle dans $\boldsymbol{R}^{n}, \mu=0$ dès que $N * \mu$ a un sens et $N * \mu=0$. 
Dans ce cas, la signe + signifie la somme vectorielle dans $\boldsymbol{R}^{n}$. Voyons le lemme 1. Pour toute $f$ de $C_{K}^{+}$et pour toute $g$ de $C_{K}^{+}$à $\operatorname{supp}(g) \subset\left\{x \in \boldsymbol{R}^{n} ; N_{2} * f(x)>0\right\}$. Alors il existe une constante $c_{g}>0$ telle que $N_{1} * g(x) \leqq c_{g} N_{2} * f(x)$ sur supp $(g)$, et par suite cette inégalité a lieu sur $\boldsymbol{R}^{n}$, d'où $\operatorname{supp}\left(N_{1}\right)+\operatorname{supp}(g)=\operatorname{supp}\left(N_{1} * g\right) \subset \operatorname{supp}\left(N_{2} * f\right)$. La fonction $g$ étant quelconque, on obtient $\operatorname{supp}\left(N_{1}\right)+\operatorname{supp}\left(N_{2} * f\right)$ $\subset \operatorname{supp}\left(N_{2} * f\right)$. La fonction $f$ étant aussi quelconque, on obtient $\operatorname{supp}\left(N_{1}\right)$ $+\operatorname{supp}\left(N_{2}\right) \subset \operatorname{supp}\left(N_{2}\right)$, d'où le lemme 1 .

Dans le présent lemme, si $\operatorname{supp}\left(N_{2}\right) \ni 0$, alors $\operatorname{supp}\left(N_{1}\right) \subset \operatorname{supp}\left(N_{2}\right)$.

CoRollaire 1. Soient $N_{0}$ un noyau de convolution de Hunt sur $\boldsymbol{R}^{n}$ et $N$ un noyau de convolution sur $\boldsymbol{R}^{n} \grave{a} \operatorname{supp}(N) \ni 0$. S'il existe une mesure positive $\mu$ dans $\boldsymbol{R}^{n}$ telle que $N=N_{0} * \mu$, alors $\operatorname{supp}\left(N_{0}\right) \subset \operatorname{supp}(N)$.

En effet, on connaît bien que $N_{0}$ satisfait au principe de domination (c'est-à-dire, au principe de domination relatif à lui-même), et donc $N_{0}$ satisfait au principe de domination relatif au noyau $N$, d'où $\operatorname{supp}\left(N_{0}\right)$ $\subset \operatorname{supp}(N)$.

De la même manière que dans $\S 2$, on pose, pour une constante réelle $a$,

$$
\kappa_{a}= \begin{cases}\exp (-a t) d t & \operatorname{sur} \boldsymbol{R}^{+} \\ 0 & \text { dans } \boldsymbol{R}^{1}-\boldsymbol{R}^{+} .\end{cases}
$$

Alors $\kappa_{a}$ est un unique noyau de convolution de Hunt sur $\boldsymbol{R}^{1}$ tel que, au sens des distributions, $\left(\frac{d}{d t}+a\right) \kappa_{a}=\varepsilon$.

LEMME 2. Soient a une constante réelle et $N$ un noyau de convolution sur $\boldsymbol{R}^{1}$. Alors les trois énoncés suivants sont équivalents:

(1) Pour tout l'entier $m \geqq 0,(-1)^{m}\left(\frac{d}{d t}+a\right)^{m} N$ est de $C P\left(\boldsymbol{R}^{1}\right)$ et $N$ satisfait au principle de domination relatif au noyau $\kappa_{a}$.

(2) $N$ est de la forme

$$
N=c \varepsilon+\int \kappa_{p} d \nu(p)
$$

où $c \in \boldsymbol{R}^{+}$et $\nu$ est une mesure positive sur $[a,+\infty) \grave{a} \int_{a+1}^{\infty} \frac{1}{p} d \nu(p)$ $<+\infty$. 
(3) Il existe la classe divisible $\left(N_{\tilde{p}}\right)_{\tilde{p} \in \underset{m \geqq 1}{\cup}\left(\boldsymbol{R}^{+)^{m}}\right.}$ associée au noyau $N$ relativement au noyau $\kappa_{a}$.

Montrons d'abord l'implication $(1) \Rightarrow(2)$. D'après le lemme 1 , on a $\operatorname{supp}(N) \subset \operatorname{supp}\left(\kappa_{a}\right)=\boldsymbol{R}^{+}$. Soit $N_{a}$ le noyau de convolution sur $\boldsymbol{R}^{1}$ défini par $d N_{a}=\exp (a t) d N(t)$. Alors, pour tout l'entier $m \geqq 0, \frac{d^{m}}{d t^{m}} N_{a}$ $={ }_{\mathrm{e}}^{\infty} \exp (a t)\left(\frac{d}{d t}+a\right)^{m} N$. Donc, d'après la proposition 5 , il existe uniquement une constante $c \geqq 0$ et une mesure positive $\nu^{\prime}$ sur $R^{+}$à $\int_{1}^{\infty} \frac{1}{p} d \nu^{\prime}(p)$ $<+\infty$ telles que $N_{a}=c \varepsilon+\int \kappa_{p} d \nu^{\prime}(p)$. Soit $\nu$ la mesure positive obtenue de $\nu^{\prime}$ par la translation de $a$; alors on obtient que $N$ est de la forme dans (2).

Montrons ensuite l'implication (2) $\Rightarrow(3)$. Il est déjà connu, dans [6], que pour une constante $c \geqq 0$ et pour une mesure positive $\nu$ sur $\boldsymbol{R}^{+}$à $\int_{1}^{\infty} \frac{1}{p} d \nu(p)<+\infty$, il existe uniquement une constante $c^{\prime} \geqq 0$ et une mesure positive $\nu^{\prime}$ sur $R^{+}$à $\int_{1}^{\infty} \frac{1}{p} d \nu^{\prime}(p)<+\infty$ telles que

$$
\left(c \varepsilon+\int \kappa_{p} d \nu(p)\right) *\left(c^{\prime} \varepsilon+\int \kappa_{p} d \nu^{\prime}(p)\right)=\kappa
$$

On connaît aussi, d'après [6], que pour les mêmes $c, \nu$ que ci-dessus, il existe la résolvante $\left(\tilde{N}_{p}\right)_{p \geqq 0}$ associée au noyau $c \varepsilon+\int \kappa_{p} d \nu(p)$ et que $\tilde{N}_{p}$ est de la forme $\tilde{N}_{p}=c_{p} \varepsilon+\int \kappa_{q} d \nu_{p}(q)(\forall p \geqq 0)$, où $c_{p} \in R^{+}$et $\nu_{p} \in M^{+}\left(R^{+}\right)$ à $\int_{1}^{\infty} \frac{1}{q} d \nu_{p}(q)<+\infty$.

Soit $N=c \varepsilon+\int \kappa_{p} d \nu(p)$ le noyau de convolution sur $\boldsymbol{R}^{1}$ donné dans (2), et posons $N_{a}=c \varepsilon+\int \kappa_{p} d \tau_{-a^{\nu}}(p)$, où $\tau_{-a^{\nu}}$ est la mesure positive obtenue de $\nu$ par la translation de $-a$. Alors il existe la classe divisible $\left(N_{a, \tilde{p}}\right)_{\tilde{p} \in \underset{m \geqq 1}{\cup}\left(\boldsymbol{R}^{+}\right) m}$ associée au noyau $N_{a}$ relativement au noyau $\kappa$, et pour tout $\tilde{p}, N_{a, \tilde{p}}$ est de la forme $N_{a, \tilde{p}}=c_{a, \tilde{p}} \varepsilon+\int \kappa_{p} d \nu_{a, \tilde{p}}(p)$, où $c_{a, \tilde{p}} \in \boldsymbol{R}^{+}$et 
$\nu_{a, \tilde{p}} \in M^{+}\left(\boldsymbol{R}^{+}\right)$à $\int_{1}^{\infty} \frac{1}{p} d \nu_{a, \tilde{p}}(p)<+\infty$. Soit $\nu_{\tilde{p}}$ la mesure positive obtenue de $\nu_{a, \tilde{p}}$ par la translation de $a$ et posons

$$
N_{\tilde{p}}=c_{\tilde{p} \varepsilon}+\int \kappa_{p} d \nu_{\tilde{p}}(p)
$$

où $c_{\tilde{p}}=c_{a, \tilde{p}}$. Alors on peut montrer facilement que $\left(N_{\tilde{p}}\right)_{\tilde{p} \in \underset{m \geqq 1}{\cup}\left(R^{+}\right)^{m}}$ est la classe divisible associée au noyau $N$ relativement au noyau $\kappa_{a}$.

Montrons finalement l'implication (3) $\Rightarrow(1)$. Pour tout $\tilde{p}=\left(p_{1}, \cdots, p_{m}\right)$ de $\bigcup_{m \geqq 1}\left(\boldsymbol{R}^{+}\right)^{m}$, on a d'abord, d'après la proposition 6 et le corollaire 1 , $\operatorname{supp}\left(N_{\tilde{p}}\right) \subset \operatorname{supp}\left(\kappa_{a}\right)=\boldsymbol{R}^{+} . \quad$ On a ensuite

$$
\left(\left(\frac{d}{d t}+a\right) N_{\tilde{p}}\right) * N_{\left(p_{1}, \cdots, p_{m}, 0\right)}=\varepsilon
$$

et

$$
\lim _{p \rightarrow \infty} p\left(\varepsilon-p N_{\left(p_{1}, \cdots, p_{m}, p\right)}\right) * N_{\left(p_{1}, \cdot, p_{m}, 0\right)}=\varepsilon
$$

vaguement dans $\boldsymbol{R}^{1}$, car $N_{\left(p_{1}, \cdots, p_{m}, 0\right)}$ est un noyau de convolution de Hunt sur $\boldsymbol{R}^{1}$. Donc on peut montrer facilement que $\left(p\left(\varepsilon-p N_{\left(p_{1}, \cdots, p_{m}, p\right)}\right)\right)_{p>0}$ converge vers $\left(\frac{d}{d t}+a\right) N_{\tilde{p}}$ au sens des distributions dans $R^{1}$ lorsque $p \rightarrow+\infty$. Par conséquent, $(-1)\left(\frac{d}{d t}+a\right) N_{\tilde{p}}$ est de $C P\left(\boldsymbol{R}^{1}\right)$.

Soit $k$ un entier $\geqq 1$ et supposons que, quel que soit $\tilde{p}$ de $\bigcup_{m \geqq 1}\left(\boldsymbol{R}^{+}\right)^{m}$, $(-1)^{k}\left(\frac{d}{d t}+a\right)^{k} N_{\tilde{p}}$ est de $C P\left(\boldsymbol{R}^{1}\right) . \quad$ Pour tout $\tilde{p}=\left(p_{1}, \cdots, p_{m}\right)$ de $\bigcup_{m \geqq 1}\left(\boldsymbol{R}^{+}\right)^{m}$, on a

$$
\lim _{p \rightarrow \infty} p\left(p N_{\left(p_{1}, \cdots, p_{m}, p\right)}-\varepsilon\right)=(-1)\left(-\frac{d}{d t}+a\right) N_{\tilde{p}}
$$

au sens des distributions dans $\boldsymbol{R}^{1}$, et par suite on obtient la famille

$$
\left(p(-1)^{k}\left(\frac{d}{d t}+a\right)^{k} N_{\left(p_{1}, \cdots, p_{m}, p\right)}-(-1)^{k}\left(\frac{d}{d t}+a\right)^{k} \varepsilon\right)_{p>0}
$$

converge vers $(-1)^{k+1}\left(\frac{d}{d t}+a\right)^{k+1} N_{\tilde{p}}$ au sens des distributions dans $\boldsymbol{R}^{1}$ 
lorsque $p \rightarrow+\infty$. D'après notre hypothèse, $(-1)^{k+1}\left(\frac{d}{d t}+a\right)^{k+1} N_{\tilde{p}}$ est de $C P\left(R^{1}\right)$, et par suite on obtient, par récurrence, $(3) \Rightarrow(1)$.

Considérons ensuite un opérateur différentiel elliptique d'ordre $\leqq 2$ sur $\boldsymbol{R}^{n}$ à coéfficients constants. On dit, d'après [6] et [7], qu'un noyau de convolution $N$ sur $\boldsymbol{R}^{n}$ est complètement $L$-sous-harmonique si, pour tout l'entier $m \geqq 0, L^{m} N$ est de $C P\left(\boldsymbol{R}^{n}\right)$, où $L^{0} N=N, L^{1}=L$ et $L^{m+1}$ $=L^{m} L$.

LEMME 3. Soient $L=\sum_{i=1}^{n} \sum_{j=1}^{n} a_{i j} \frac{\partial^{2}}{\partial x_{i} \partial x_{j}}+\sum_{i=1}^{n} b_{i} \frac{\partial}{\partial x_{i}}+c$ un opérateur différentiel elliptique sur $\boldsymbol{R}^{n}$ à coéfficients constants, $\tilde{a}=\left(\tilde{a}_{1}, \tilde{a}_{2}, \cdots, \tilde{a}_{n}\right)$ un point fixé de $\boldsymbol{R}^{n}$ et $N$ un noyau de convolution sur $\boldsymbol{R}^{n}$. On désigne par $N^{(\tilde{a})}$ le noyau de convolution sur $\boldsymbol{R}^{n}$ défini par $d N^{(\tilde{a})}=\exp (-\tilde{a} \cdot x) d N(x)$. Si $N$ est complètement L-sous-harmonique, alors $N^{(\tilde{a})}$ est aussi complètement $L_{\tilde{a}^{-}}$sous-harmonique, où

$$
L_{\tilde{a}}=\sum_{i=1}^{n} \sum_{j=1}^{n} a_{i j}\left(\frac{\partial}{\partial x_{i}}+\tilde{a}_{i}\right)\left(\frac{\partial}{\partial x_{j}}+\tilde{a}_{j}\right)+\sum_{i=1}^{n} b_{i}\left(\frac{\partial}{\partial x_{i}}+\tilde{a}_{i}\right)+c .
$$

En effet, on a, pour tout $i$ avec $1 \leqq i \leqq n$,

$$
\left(\frac{\partial}{\partial x_{i}}+\tilde{a}_{i}\right) N^{(\tilde{a})}=\exp (-\tilde{a} \cdot x) \frac{\partial}{\partial x_{i}} N
$$

au sens des distributions dans $\boldsymbol{R}^{n}$, et donc on peut montrer, par récurrence, $N^{\tilde{a}}$ est complètement $L_{\tilde{a} \text {-sous-harmonique. }}$

On notera $S_{1,0}$ la sphère d'unité de centre 0 .

LEMME 4. Soient a une constante $\geqq 0$ et $L=\Delta-a$, où $\Delta$ est le laplacien ordinaire sur $\boldsymbol{R}^{n}$. Si un noyau de convolution $N$ sur $\boldsymbol{R}^{n}$ est de masse totale finie et complètement $L$-sous-harmonique, alors il existe uniquement une constante $c \geqq 0$ et deux familles $\left(\alpha_{\sigma}^{(1)}\right)_{\sigma \in S_{1,0}},\left(\alpha_{\sigma}^{(2)}\right)_{\sigma \in S_{1,0}}$ de mesures positives sur $[\sqrt{a},+\infty)$ telle que la transformée de Fourier $\hat{N}$ de $N$ soit de la forme

$$
\hat{N}(x)=\hat{N}(r, \sigma)=c+\int \frac{1}{p+2 \pi \sqrt{-1} r} d \alpha_{\sigma}^{(1)}(p)+\int \frac{1}{p-2 \pi \sqrt{-1} r} d \alpha_{\sigma}^{(2)}(p) .
$$

En effet, d'après la proposition 1 , pour tout l'entier $m \geqq 0$, la distribution $(-1)^{m}\left(4 \pi^{2}|x|^{2}+a\right) \hat{N}(x) d x$ dans $\boldsymbol{R}^{n}$ est de $C T P$. Posons, pour un point $\sigma$ de $S_{1,0}$, 


$$
\psi_{\sigma}(t)=\left\{\begin{array}{ll}
\hat{N}(t, \sigma) & \text { si } t \geqq 0 \\
\hat{N}(-t,-\sigma) & \text { si } t<0
\end{array} ;\right.
$$

alors $\psi_{\sigma}$ est finie, continue et de type positif dans $\boldsymbol{R}^{1}$. En considérant une certaine rotation de $N$, on obtient que, pour tout l'entier $m \geqq 0$, $(-1)^{m}\left(4 \pi^{2}|t|^{2}+a\right)^{m} \psi_{\sigma}(t) d t$ est de $C T P\left(\boldsymbol{R}^{1}\right)$ (cf. le corollaire 1 dans [7]). Soit $\kappa_{\sigma}$ un noyau de convolution sur $\boldsymbol{R}^{1}$ de masse totale finie tel que $\hat{\kappa}_{\sigma}=\psi_{\sigma}$. En utilisant encore la proposition 1, on a, pour tout l'entier $m \geqq 0,\left(\frac{d^{2}}{d t^{2}}-a\right)^{m} \kappa_{\sigma} \in C P\left(\boldsymbol{R}^{1}\right)$, et par suite $\frac{d^{2 m}}{d t^{2 m}} \kappa_{\sigma}$ est encore de $C P\left(\boldsymbol{R}^{1}\right)$ (cf. par exemple, le lemme 5 dans [7]). Ayant $\int d \kappa_{o}<+\infty$, on obtient facilement que, pour tout l'entier $m \geqq 1, \frac{d^{2 m+1}}{d t^{2 m+1}} \kappa_{\sigma} \leqq 0$ au sens des distributions dans $(0,+\infty)$ et $\frac{d^{2 m+1}}{d t^{2 m+1}} \kappa_{\sigma} \geqq 0$ au sens des distributions dans $(-\infty, 0)$. Donc il existe une constante $c_{\sigma} \geqq 0$, une fonction sommable $k_{\sigma}(t) \geqq 0$ dans $R^{1}$ et deux fonctions infiniment derivables $\varphi_{1, \sigma} \geqq 0, \varphi_{2, \sigma} \geqq 0$ telles que $\kappa_{\sigma}=c_{\sigma} \varepsilon+k_{\sigma}(t) d t, k_{\sigma}(t)=\varphi_{1, \sigma}(t)$ presque partout (noté p.p.) dans $(0,+\infty), k_{\sigma}(t)=\varphi_{2, \sigma}(-t)$ p.p. dans $(-\infty, 0)$ et pour tout l'entier $m \geqq 0$, $(-1)^{m} \frac{d^{m}}{d t^{m}} \varphi_{i, \sigma}(t) \geqq 0$ dans $(0,+\infty) \quad(i=1,2)$. D'après le théorème de Bernstein, il existe une mesure positive $\alpha_{i, \sigma}$ sur $\boldsymbol{R}^{+}$, et une seule telle que l'on ait

$$
\varphi_{i, \sigma}(t)=\int \exp (-p t) d \alpha_{i, \sigma}(p) \quad \text { dans } \quad(0,+\infty) \quad(i=1,2) .
$$

Ayant $\int_{0}^{\infty} \varphi_{i, \sigma}(t) d t<+\infty$, on a $\alpha_{i, \sigma}(\{0\})=0$ et $\int_{0}^{\infty} \frac{1}{p} d \alpha_{i, \sigma}(p)<+\infty$. Comme

$$
(-1)^{m} \frac{d^{m}}{d t^{m}}\left(\frac{d^{2}}{d t^{2}}-a\right) \varphi_{i, \sigma}(t) \geqq 0 \quad \text { dans } \quad(0,+\infty)
$$

la mesure $\left(p^{2}-a\right) d \alpha_{i, \sigma}(p)$ doit être positive sur $\boldsymbol{R}^{+}$, d'après le théorème de Bernstein, d'où $\operatorname{supp}\left(\alpha_{i, \sigma}\right) \subset[\sqrt{a},+\infty)(i=1,2)$. On a ensuite, pour tout $r \geqq 0$,

$$
\hat{N}(r, \sigma)=\psi_{\sigma}(r)=c_{\sigma}+\int_{0}^{\infty} \int \exp (-(p+2 \pi \sqrt{-1} r) s) d \alpha_{1, \sigma}(p) d s
$$




$$
+\int_{0}^{\infty} \int \exp (-(p-2 \pi \sqrt{-1} r) s) d \alpha_{2, \sigma}(p) d s .
$$

Donc, d'après le théorème de Fubini,

$$
\hat{N}(r, \sigma)=c_{\sigma}+\int \frac{1}{p+2 \pi \sqrt{-1} r} d \alpha_{1, \sigma}(p)+\int \frac{1}{p-2 \pi \sqrt{-1} r} d \alpha_{2, \sigma}(p) .
$$

On a évidemment, pour tout $\sigma$ de $S_{1,0}, c_{\sigma}=N(\{0\})$. En posant $c=c_{\sigma}$, $\alpha_{\sigma}^{(1)}=\alpha_{1, \sigma}$ et $\alpha_{\sigma}^{(2)}=\alpha_{2, \sigma}$, on obtient une constante $c$ et deux familles $\left(\alpha_{\sigma}^{(1)}\right)_{\sigma \in S_{1}, 0},\left(\alpha_{\alpha}^{(2)}\right)_{\sigma \in S_{1}, 0}$ demandées.

Soient $c^{\prime},\left(\alpha_{\sigma}^{(3)}\right)_{\sigma \in S_{1}, 0}$ et $\left(\alpha_{\sigma}^{(4)}\right)_{\sigma \in S_{1}, 0}$ une autre constante $\geqq 0$ et deux familles de mesures positives sur $[\sqrt{a},+\infty)$ telles que

$$
\hat{N}(r, \sigma)=c^{\prime}+\int \frac{1}{p+2 \pi \sqrt{-1} r} d a_{\sigma}^{(3)}(p)+\int \frac{1}{p-2 \pi \sqrt{-1} r} d \alpha_{\sigma}^{(4)}(p) .
$$

Ayant $c^{\prime}=N(\{0\})$, on a $c=c^{\prime}$. En considérant la transformation inverse de Fourier, on a

$$
\int \exp (-p t) d \alpha_{\sigma}^{(i)}(p)=\int \exp (-p t) d \alpha_{\sigma}^{(i+2)}(p) \quad \text { dans } \quad(0,+\infty) \quad(i=1,2),
$$

et par suite, pour tout $\sigma$ de $S_{1,0}, \alpha_{\sigma}^{(1)}=\alpha_{\sigma}^{(3)}$ et $\alpha_{\sigma}^{(2)}=\alpha_{\sigma}^{(4)}$. La démonstration est ainsi complète.

Lemme 5. Soient a et $L$ les mêmes que ci-dessus; supposons qu'un noyau de convolution $N$ sur $\boldsymbol{R}^{n}$ est complètement $L$-sous-harmonique et que, pour tout l'entier $m \geqq 0, \int_{|x|>0} d\left(L^{m} N\right)<+\infty$. Soit $N^{(m)}$ le noyau de convolution sur $\boldsymbol{R}^{n}$ defini par $\int f d N^{(m)}=\int_{|x|>0} f(x) d\left(L^{m} N\right)(x)$ pour toute $f \in C_{k}$, et soient $\left(\alpha_{\sigma}^{(1)}\right)_{\sigma \in S_{1}, 0}$ et $\left(\alpha^{(2)}\right)_{\sigma \in S_{1}, 0}$ les deux familles des mesures positives sur $[\sqrt{a},+\infty)$ obtenues dans le présent lemme pour $N$. Alors, pour tout l'entier $m \geqq 0, \int \frac{\left(p^{2}-a\right)^{m}}{p} d \alpha_{\sigma}^{(1)}(p)<+\infty, \int \frac{\left(p^{2}-a\right)^{m}}{p} d \alpha_{\sigma}^{(2)}(p)<+\infty$ et

$$
\widehat{N^{(m)}}(r, \sigma)=\int \frac{\left(p^{2}-a\right)^{m}}{p+2 \pi \sqrt{-1} r} d \alpha_{\sigma}^{(1)}(p)+\int \frac{\left(p^{2}-a\right)^{m}}{p-2 \pi \sqrt{-1} r} d \alpha_{\sigma}^{(2)}(p)
$$

En effet, d'après le lemme 4,

$$
\widehat{N^{(0)}}(r, \sigma)=\int \frac{1}{p+2 \pi \sqrt{-1} r} d \alpha_{\sigma}^{(1)}(p)+\int \frac{1}{p-2 \pi \sqrt{-1} r} d \alpha_{\sigma}^{(2)}(p),
$$


$\int_{0}^{\infty} \frac{1}{p} d \alpha_{\sigma}^{(1)}(p)<+\infty$ et $\int_{0}^{\infty} \frac{1}{p} d \alpha_{\sigma}^{(2)}(p)<+\infty$. Il suffit de montrer

$$
\widehat{N^{(1)}}(r, \sigma)=\int \frac{p^{2}-a}{p+2 \pi \sqrt{-1} r} d \alpha_{\sigma}^{(1)}(p)+\int \frac{p^{2}-a}{p-2 \pi \sqrt{-1} r} d \alpha_{\sigma}^{(2)}(p),
$$

car si c'est vrai, il résulte de l'inégalité $\int d N^{(1)}<+\infty$ et du lemme 4 que, pour tout $\sigma$ de $S_{1,0}, \int_{0}^{\infty} \frac{p^{2}-\alpha}{p} d\left(\alpha_{\sigma}^{(1)}+\alpha_{\sigma}^{(2)}\right)(p)<+\infty$, et on peut montrer, par récurrence, notre lemme. On a

$$
\begin{aligned}
\widehat{L N}^{(0)}(r, \sigma)= & \int\left(\frac{p^{2}-a}{p+2 \pi \sqrt{-1} r}-p+2 \pi \sqrt{-1} r\right) d \alpha_{\sigma}^{(1)}(p) \\
& +\int\left(\frac{p^{2}-a}{p-2 \pi \sqrt{-1} r}-p-2 \pi \sqrt{-1} r\right) d \alpha_{\sigma}^{(2)}(p) .
\end{aligned}
$$

Posons, pour un point $\sigma$ de $S_{1,0}$,

$$
\varphi_{\sigma}(t)=\left\{\begin{array}{ll}
\widehat{L N}^{(0)}(t, \sigma) & \text { si } t \geqq 0 \\
\widehat{L N}^{(0)}(-t,-\sigma) & \text { si } t<0
\end{array} ;\right.
$$

alors $\varphi_{\sigma}(t) d t$ est de $C T P\left(\boldsymbol{R}^{1}\right)$. Soit $u_{\sigma}$ la distribution dans $\boldsymbol{R}^{1}$ telle que $\hat{u}_{\sigma}=\varphi_{\sigma}(t) d t$ (cf. la proposition 1). On a alors

$$
u_{\sigma}=\left\{\begin{array}{lll}
\left(\int \exp (-p t)\left(p^{2}-a\right) d \alpha_{\sigma}^{(1)}(p)\right) d t & \text { dans } & (0,+\infty) \\
\left(\int \exp (p t)\left(p^{2}-a\right) d \alpha_{\sigma}^{(2)}(p)\right) d t & \text { dans } & (-\infty, 0)
\end{array}\right.
$$

D'autre part, $N^{(1)}$ étant complètement $L$-sous-harmonique et ayant $\int d N^{(1)}$ $<+\infty$ et $N^{(1)}(\{0\})=0$, on obtient, d'après le lemme 4, qu'il existe uniquement deux familles $\left(\alpha_{\sigma, 1}^{(1)}\right)_{\sigma \in S_{1,0}}$ et $\left(\alpha_{\sigma, 1}^{(2)}\right)_{\sigma \in S_{1,0}}$ de mesures positives sur $[\sqrt{a},+\infty)$ telles que

$$
\widehat{N^{(1)}}(r, \sigma)=\int \frac{1}{p+2 \pi \sqrt{-1} r} d \alpha_{\sigma, 1}^{(1)}(p)+\int \frac{1}{p-2 \pi \sqrt{-1} r} d \alpha_{\sigma, 1}^{(2)}(p) .
$$

Dans ce cas, $\alpha_{\sigma, 1}^{(1)}(\{0\})=0, \alpha_{\sigma, 1}^{(2)}(\{0\})=0$ et $\int_{0}^{\infty} \frac{1}{p} d\left(\alpha_{\sigma, 1}^{(1)}+\alpha_{\sigma, 1}^{(2)}\right)(p)<+\infty$. Soit $\kappa_{\sigma, 1}$ le noyau de convolution sur $R^{1}$ de masse totale finie tel que $\widehat{\kappa_{\sigma, 1}}(t)=\widehat{N^{(1)}}(t, \sigma)$ sur $\boldsymbol{R}^{+}$et $\widehat{\kappa_{\sigma, 1}}(t)=\widehat{N^{(1)}}(-t,-\sigma)$ dans $\boldsymbol{R}^{1}-\boldsymbol{R}^{+}$. Ayant 
$\operatorname{supp}\left(L N^{(0)}-N^{(1)}\right) \subset\{0\}$, on obtient que $L \widehat{N}^{(0)}-\widehat{N}^{(1)}$ est égale à un polynôme, et donc $\varphi_{\sigma}-\widehat{\kappa_{\sigma, 1}}$ est aussi égale à un polynôme. Par conséquent, $u_{\sigma}=\kappa_{\sigma, 1}$ en dehors de l'origine, et par suite

$$
\int \exp (-p t)\left(p^{2}-a\right) d \alpha_{\sigma}^{(i)}(p)=\int \exp (-p t) d \alpha_{\sigma, 1}^{(i)}(p)
$$

dans $(0,+\infty) \quad(i=1,2)$, d'où $d \alpha_{\sigma, 1}^{(i)}=\left(p^{2}-a\right) d \alpha_{\sigma}^{(i)}(p) \quad(i=1,2)$, d'après l'injectivité de la transformation de Laplace.

De la même manière que ci-dessus, si $N$ est complètement $L$-sousharmonique et s'il existe un entier $k \geqq 1$ tel que, pour tout l'entire $m$ à $1 \leqq m \leqq k, \int_{|x|>0} d\left(L^{m} N\right)<+\infty$, alors $N^{(m)}$ est de la forme que ci-dessus $(1 \leqq \forall m \leqq k)$.

LEMME 6. Soient a une constante $\geqq 0$ et $\alpha_{1}, \alpha_{2}$ deux mesures positives sur $[\sqrt{a},+\infty)$ telles que $\alpha_{1}(\{0\})=\alpha_{2}(\{0\})=0$ et pour tout l'entier $m \geqq 0, \int\left(p+\frac{1}{p}\right)^{m} d\left(\alpha_{1}+\alpha_{2}\right)(p)<+\infty$. Posons, pour tout l'entier $m \geqq 0$,

$$
\lambda_{m}(t)=\int \frac{\left(p^{2}-a\right)^{m}}{p+2 \pi \sqrt{-1} t} d \alpha_{1}(p)+\int \frac{\left(p^{2}-a\right)^{m}}{p-2 \pi \sqrt{-1} t} d \alpha_{2}(p),
$$

où l'on suppose ici $\left(p^{2}-a\right)^{0}=1$ pour tout $p$. Si, pour tout l'entier $m \geqq 0,\left(4 \pi^{2}|t|^{2}+a\right) \lambda_{m}(t)$ est bornée sur $\boldsymbol{R}^{1}$, alors $\alpha_{1}=\alpha_{2}$.

En effet, on a

$$
\begin{aligned}
-\left(4 \pi^{2}|t|^{2}+a\right) \lambda_{m}(t)= & \int \frac{\left(p^{2}-a\right)^{m+1}}{p+2 \pi \sqrt{-1} t} d \alpha_{1}(p)+\int \frac{\left(p^{2}-a\right)^{m+1}}{p-2 \pi \sqrt{-1} t} d \alpha_{2}(p) \\
& -\int p\left(p^{2}-a\right)^{m} d\left(\alpha_{1}+\alpha_{2}\right)(p) \\
& +2 \pi \sqrt{-1} t \int\left(p^{2}-a\right)^{m} d\left(\alpha_{1}-\alpha_{2}\right)(p),
\end{aligned}
$$

et par suite $\int\left(p^{2}-a\right)^{m} d \alpha_{1}(p)=\int\left(p^{2}-a\right)^{m} d \alpha_{2}(p)$. Donc, pour tout l'entier $m \geqq 0, \int p^{2 m} d \alpha_{1}(p)=\int p^{2 m} d \alpha_{2}(p)<+\infty$. Par conséquent, pour tout $t$ de $\boldsymbol{R}^{+}$

$$
\int \exp \left(-p^{2} t\right) d \alpha_{1}(p)=\int \exp \left(-p^{2} t\right) d \alpha_{2}(p)
$$


d'où $\alpha_{1}=\alpha_{2}$.

Pour considérer le noyau de convolution divisible, on définit, pour tout l'entier $m \geqq 2$, une famille $\left(d_{i}^{(m)}\right)_{i=1}^{m-1}$ de fonctions positives, finies et continues dans In. $\left(\boldsymbol{R}^{+}\right)^{m}$, où In. $\left(\boldsymbol{R}^{+}\right)^{m}$ désigne l'intérieur de $\left(\boldsymbol{R}^{+}\right)^{m}$ dans $\boldsymbol{R}^{m}$. On pose $d_{1}^{(2)}\left(t_{1}, t_{2}\right)=t_{1}$ dans In. $\left(\boldsymbol{R}^{+}\right)^{2}$. Supposons que, pour un entier $m \geqq 2$, $\left(d_{i}^{(m)}\right)_{i=1}^{m-1}$ est définie. Soit $\left(t_{1}, \cdots, t_{m+1}\right)$ un point quelconque dans In. $\left(\boldsymbol{R}^{+}\right)^{m+1}$; alors on pose, pour tout l'entier $i$ à $1 \leqq i \leqq m$,

$$
\begin{aligned}
d_{i}^{(m+1)}\left(t_{1}, \cdots, t_{m+1}\right)= & \frac{t_{1} \cdots t_{m+1}}{t_{2} \cdots t_{i+1}} \\
& +t_{1}^{2} \sum_{j=1}^{i} d_{j}^{(m)}\left(t_{2}, \cdots, t_{m+1}\right) \frac{t_{j+1} \cdots t_{m+1}}{\left(t_{j+1} \cdots t_{m+1}\right)\left(t_{j+1} \cdots t_{i+1}\right)},
\end{aligned}
$$

où l'on suppose $d_{m}^{(m)}=0$. On obtient ainsi une famille $\left(\left(d_{i}^{(m)}\right)_{i=1}^{m-1}\right)_{m=2}^{\infty}$.

LEMme 7. Soient $\tilde{N}$ un noyau de convolution de Hunt borné sur $\boldsymbol{R}^{n}$ et $\tilde{u}$ la laplacien généralisé sur $\boldsymbol{R}^{n}$ vérifiant $\tilde{u} * \tilde{N}=-\varepsilon$. Supposons que, pour un noyau de convolution donné $N$ sur $\boldsymbol{R}^{n}$, il existe la classe divisible $\left(N_{\tilde{p}}\right)_{\tilde{p} \in \underset{m \geqq 1}{\cup}(\boldsymbol{R}+)^{m}}$ associée au noyau $N$ relativement au noyau $\tilde{N}$ et posons, pour un point $\tilde{p}=\left(p_{1}, \cdots, p_{m}\right)$ de $\bigcup_{m \geqq 2} \operatorname{In} .\left(\boldsymbol{R}^{+}\right)^{m}$,

$$
\begin{aligned}
M_{\tilde{p}}= & \left.p_{1} \cdots p_{m} N * N_{\left(0, p_{1}\right)} * \cdots * N_{\left(0, p_{1}, \cdots, p_{m}\right.}\right) \\
& +\sum_{i=1}^{m-1} d_{i}^{(m)}\left(p_{1}, \cdots, p_{m}\right) N_{\left(0, p_{1}, \cdots, p_{i}\right)} * \cdots * N_{\left(0, p_{1}, \cdots, p_{m}\right)} .
\end{aligned}
$$

Alors

$$
\begin{aligned}
\tilde{u} * M_{\tilde{p}}= & p_{1}^{2}\left(p_{2} \cdots p_{m} N_{\left(0, p_{1}\right)} * \cdots * N_{\left(0, p_{1}, \cdots, p_{m}\right)}\right. \\
& \left.+\sum_{i=1}^{m-2} d_{i}^{(m-1)}\left(p_{2}, \cdots, p_{m}\right) N_{\left(0, p_{1}, \cdots, p_{i+1}\right)} * \cdots * N_{\left(0, p_{1}, \cdots, p_{m}\right)}\right)
\end{aligned}
$$

en dehors de l'origine.

En effet, on remarque d'abord que, pour tout $\tilde{p}=\left(p_{1}, \cdots, p_{m}\right)$ de $\bigcup_{m \geqq 1}\left(\boldsymbol{R}^{+}\right)^{m}$, d'après $N_{\tilde{p}} * N_{\left(p_{1}, \cdots, p_{m}, 0\right)}=\tilde{N}, N_{\tilde{p}}$ est aussi un noyau de convolution de Hunt borné sur $\boldsymbol{R}^{n}$. On a donc, pour tout $i$ à $1 \leqq i \leqq m$, $p_{i} \int d N_{\left(0, p_{1}, \ldots, p_{i}\right)} \leqq 1$, et par suite $M_{\tilde{p}}$ définit un noyau de convolution borné sur $R^{n}$, d'où $\tilde{u} * M_{\tilde{p}}$ est définie. Ayant

$$
N_{\left(0, p_{1}, \ldots, p_{i}\right)} * N_{\left(0, p_{1}, \ldots, p_{i+1}\right)}=\tilde{N} *\left(\varepsilon-p_{i+1} N_{\left(0, p_{1}, \ldots, p_{i+1}\right)}\right),
$$


où dans le cas où $i=0, N_{\left(0, p_{1}, \ldots, p_{i}\right)}$ signifie $N$, on obtient donc

$$
\begin{aligned}
\tilde{u} * M_{\tilde{p}}= & p_{1} \cdots p_{m}\left(\left(p_{1} N_{\left(0, p_{1}\right)}-\varepsilon\right) * N_{\left(0, p_{1}, p_{2}\right)} * \cdots * N_{\left(0, p_{1}, \cdots, p_{m}\right)}\right) \\
& +\sum_{i=1}^{m-1} d_{i}^{(m)}\left(p_{1}, \cdots, p_{m}\right)\left(\left(p_{i+1} N_{\left(0, p_{1}, \cdots, p_{i+1}\right)}-\varepsilon\right) * N_{\left(0, p_{1}, \cdots, p_{i+2}\right)}\right. \\
& \left.* \cdots * N_{\left(0, p_{1}, \cdots, p_{m}\right)}\right) \\
= & p_{1}^{2}\left(p_{2} \cdots p_{m} N_{\left(0, p_{1}\right)} * \cdots * N_{\left(0, p_{1}, \cdots, p_{m}\right)}\right. \\
& +\frac{1}{p_{1}^{2}}\left(d_{1}^{(m)}\left(p_{1}, \cdots, p_{m}\right) p_{2}-p_{1} \cdots p_{m}\right) N_{\left(0, p_{1}, p_{2}\right)} * \cdots * N_{\left(0, p_{1}, \cdots, p_{m}\right)} \\
& +\frac{1}{p_{1}^{2}} \sum_{i=2}^{m-1}\left(d_{i}^{(m)}\left(p_{1}, \cdots, p_{m}\right) p_{i+1}-d_{i-1}^{(m)}\left(p_{1}, \cdots, p_{m}\right)\right) N_{\left(0, p_{1}, \cdots, p_{i+1}\right)} \\
& \left.* \cdots * N_{\left(0, p_{1}, \cdots, p_{m}\right)}\right)-d_{m-1}^{(m)}\left(p_{1}, \cdots, p_{m}\right) \varepsilon .
\end{aligned}
$$

D'après la définition de $\left(\left(d_{i}^{(m)}\right)_{i=1}^{m-1}\right)_{m=2}^{\infty}$, on a

$$
d_{1}^{(m)}\left(p_{1}, \cdots, p_{m}\right) p_{2}-p_{1} \cdots p_{m}=p_{1}^{2} d_{1}^{(m-1)}\left(p_{2}, \cdots, p_{m}\right)
$$

et pour tout l'entier $i$ à $2 \leqq i \leqq m-1$,

$$
d_{i}^{(m)}\left(p_{1}, \cdots, p_{m}\right) p_{i+1}-d_{i-1}^{(m)}\left(p_{1}, \cdots, p_{m}\right)=p_{1}^{2} d_{i}^{(m-1)}\left(p_{2}, \cdots, p_{m}\right) .
$$

Par conséquent on obtient immédiatement notre lemme.

On dira que $\left(\left(d_{i}^{(m)}\right)_{i=1}^{m-1}\right)_{m=2}^{\infty}$ est la famille de fonctions associée à la classe divisible. D'après le lemme $7, d_{i}^{(m)}\left(p_{1}, \cdots, p_{m}\right)$ est égale à la constante positive $c_{i}\left(\left(p_{j}\right)_{j=1}^{m}\right)$, qui a été déjà introduite dans [7].

LEMME 8. Pour tout $\left(t_{1}, \cdots, t_{m}\right)$ de $\bigcup_{m \geqq 2}$ In. $\left(\boldsymbol{R}^{+}\right)^{m}$ et pour tout l'entier $i \grave{a} 1 \leqq i \leqq m$,

$$
\lim _{t \rightarrow+\infty} \frac{1}{t} d_{i}^{(m+1)}\left(t_{1}, \cdots, t_{m}, t\right)=d_{i}^{(m)}\left(t_{1}, \cdots, t_{m}\right) .
$$

Cela est immédiatement obtenu par récurrence. On remarque ici que si, pour un noyau de convolution de Hunt $\tilde{N}$ sur $\boldsymbol{R}^{n}$ et pour un noyau de convolution $N$ sur $\boldsymbol{R}^{n}$, il existe la classe divisible $\left(N_{\tilde{p}}\right)_{\tilde{p} \in \underset{m \geqq 1}{\cup}\left(\boldsymbol{R}^{+}\right)^{m}}$ associée au noyau $N$ relativement au noyau $\tilde{N}$, alors pour un point $\tilde{p}=\left(p_{1}, \cdots, p_{m}\right)$ de $\bigcup_{m \geqq 1}\left(\boldsymbol{R}^{+}\right)^{m}$, en posant

$$
N_{\tilde{p}, \tilde{q}}=N_{\left(p_{1}, \cdots, p_{m}+q_{1}, q_{2}, \cdots, q_{k}\right)}\left(\forall \tilde{q}=\left(q_{1}, \cdots, q_{k}\right) \in \bigcup_{m \geqq 1}\left(\boldsymbol{R}^{+}\right)^{m}\right),
$$

$\left(N_{\tilde{p}, \tilde{q}}\right)_{\tilde{q} \in \underbrace{\cup}_{m \geqq 1}\left(R^{+}\right)^{m}}$ est aussi la classe divisible associée au noyau $N_{\tilde{p}}$ relativement au noyau $\tilde{N}$. 
Rappelons finalement la définition du cone convexe divisible relatif au noyau de convolution de Hunt donné.

Lemme 9. Soient $\tilde{N}$ un noyau de convolution de Hunt sur $\boldsymbol{R}^{n}$ et $C_{R}(\tilde{N})$ un cône convexe divisible relatif au noyau $\tilde{N}$. Alors, pout tout $N \neq 0$ de $C_{R}(\tilde{N})$, il existe la classe divisible associée au noyau $N$ relativement au noyau $\tilde{N}$ et elle est contenue dans $C_{R}(\tilde{N})$.

Cela résulte du fait que, pour tout $N \neq 0$ de $C_{R}(\tilde{N})$, la résolvante associée au noyau $N$ est contenue dans $C_{R}(\tilde{N})$ et de la condition (b) dans $\S 1$. Voir [6] et [7].

\section{§4. Notre théorème principal}

Rappelons notre théorème principal qui a été introduit dans $\S 1$.

THÉORÈme 1. Soit $L$ un opérateur différential elliptique d'ordre $\leqq 2$ sur $\boldsymbol{R}^{n} \grave{a}$ coéfficients constants tel qu'il existe le noyau de convolution de Hunt $G_{L}$ sur $\boldsymbol{R}^{n}$ vérifiant $L G_{L}=-\varepsilon$. Alors un cône convexe divisible relatif au noyau $G_{L}$ est uniquement déterminé et cela est égal à

$$
\left\{c \varepsilon+\int G_{L, p} d \nu(p) ; c \in \boldsymbol{R}^{+}, \nu \in M^{+}\left(\boldsymbol{R}^{+}\right) \grave{a} \int_{1}^{\infty} \frac{1}{p} d \nu(p)<+\infty\right\},
$$

où $\left(G_{L, p}\right)_{p \geq 0}$ est la résolvante associée au noyau $G_{L}$.

On connaît déjà que l'ensemble $\tilde{C}_{R}\left(G_{L}\right)=\left\{c \varepsilon+\int G_{L, p} d \nu(p) ; c \in \boldsymbol{R}^{+}\right.$, $\nu \in M^{+}\left(\boldsymbol{R}^{+}\right)$à $\left.\int_{1}^{\infty} \frac{1}{p} d \nu(p)<+\infty\right\}$ est le plus petit cône convexe divisible relatif au noyau $G_{L}$ (cf. [6] et [7]). Par conséquent, le théorème 1 résulte immédiatement du lemme 9 et du théorème suivant:

THÉORÈme 2. Soient $L, G_{L}$ les mêmes que ci-dessus et $N$ un noyau de convolution sur $\boldsymbol{R}^{n}$. Alors les deux énoncés suivants sont équivalents:

(1) Il existe la classe divisible associée au noyau $N$ relativement au noyau $G_{L}$.

(2) $N$ est de la forme

$$
N=c \varepsilon+\int G_{L, p} d \nu(p)
$$


ò̀ $c \in \boldsymbol{R}^{+}$et $\nu \in M^{+}\left(\boldsymbol{R}^{+}\right) \grave{a} \int_{1}^{\infty} \frac{1}{p} d \nu(p)<+\infty$.

Démonstration. L'implication $(2) \Rightarrow(1)$ est déjà connue, d'après le lemme 9, car $N \in \tilde{C}_{R}\left(G_{L}\right)$. Donc on montrera seulement l'implication (1) $\Rightarrow(2)$. On désigne par $\left(N_{\tilde{p}}\right)_{\tilde{p} \in \underset{m \geqq 1}{\cup}\left(R^{+}\right) m}$ la classe divisible associée au noyau $N$ relativement au noyau $G_{L}$. Alors, pour tout $\tilde{p} \in \bigcup_{m \geqq 1}\left(\boldsymbol{R}^{+}\right)^{m}, N_{\tilde{p}}$ est un noyau de convolution de Hunt sur $\boldsymbol{R}^{n}$. On remarque d'abord que, pour une mesure positive $\nu$ sur $\boldsymbol{R}^{+}, \int G_{L, p} d \nu(p)$ définisse un noyau de convolution sur $\boldsymbol{R}^{n}$ si et seulement si $\int_{1}^{\infty} \frac{1}{p} d \nu(p)<+\infty$. Cela est obtenu dans [7] dans le cas où $L$ est auto-adjoint. Le cas général peut être montré aussi de la même manière, $\operatorname{car}\left(p G_{L, p}\right)_{p>0}$ converge vaguement vers $\varepsilon$ lorsque $p \rightarrow+\infty$.

Notons $L=\sum_{i=1}^{n} \sum_{j=1}^{n} a_{i j} \frac{\partial^{2}}{\partial x_{i} \partial x_{j}}+\sum_{i=1}^{n} b_{i} \frac{\partial}{\partial x_{i}}+c$; alors, d'après l'existence de $G_{L}$, on a, pour tout $x=\left(x_{1}, \cdots, x_{n}\right)$ de $\boldsymbol{R}^{n}, \sum_{i=1}^{n} \sum_{j=1}^{n} a_{i j} x_{i} x_{j} \geqq 0$. Alors il existe une constante $a>0$, un entier $k$ à $0 \leqq k \leqq n$ et une transformation linéaire $T$ de $\boldsymbol{R}^{n}$ à lui-même à $|T|=1$ tels que $\sum_{i=1}^{n} \sum_{j=1}^{n} a_{i j} \frac{\partial^{2}}{\partial x_{i} \partial x_{j}}$ soit transformé en $a \sum_{i=1}^{k} \frac{\partial^{2}}{\partial x_{i}^{2}}$ par $T$, où $|T|$ est le déterminant de $T$. Soient $L^{(T)}, G_{L}^{(T)}$ et $N_{\tilde{p}}^{(T)}$ les transformées de $L, G_{L}$ et de $N_{\tilde{p}} \operatorname{par} T\left({ }^{\forall} p \in \bigcup_{m \geqq 1}\left(R^{+}\right)^{m}\right)$, respectivement. Alors il est facile de voir que $L^{(T)} G_{L}^{(T)}=-\varepsilon$ et $\left(N_{\tilde{p}}^{(r)}\right)_{\tilde{p} \in \underset{m \geqq 1}{\cup}\left(R^{+}\right)^{m}}$ est la classe divisible associée au noyau $N^{(T)}$ relativement au noyau $G_{L}^{(T)}$, où $N^{(T)}=N_{0}^{(T)}$. Écrivons $L^{(T)}=a \sum_{i=1}^{k} \frac{\partial^{2}}{\partial x_{i}^{2}}+\sum_{i=1}^{n} b_{i}^{\prime} \frac{\partial}{\partial x_{i}}+c^{\prime}$, et posons $\tilde{a}=\left(-\frac{b_{1}^{\prime}}{2 a}, \cdots,-\frac{b_{k}^{\prime}}{2 a}, 0, \cdots, 0\right) \in \boldsymbol{R}^{n}$. On désigne par $L_{\tilde{a}}^{(T)}$ l'opérateur différentiel défini par $L^{(T)}$ dans le lemme 3 et $\operatorname{par} G_{L}^{(T, \tilde{a})}, N^{(T, \tilde{a})}$, $N_{\tilde{p}}^{(T, \tilde{a})}$ les noyaux de convolution sur $R^{n}$ définis par $G_{L}^{(T)}, N^{(T)}$ et $N_{\tilde{p}}^{(T)}$ dans le lemme 3 , respectivement. Alors il est facile de voir que $L_{\tilde{a}}^{(T)} G_{L}^{(T, \tilde{a})}$ $=-\varepsilon$ et $\left(N_{\tilde{p}}^{(T, \tilde{a})}\right)_{\tilde{p} \in \underset{m \geqq 1}{u}\left(R^{+)^{m}}\right.}$ est la classe divisible associée au noyau $N^{(T, \tilde{a})}$ relativement au noyau $G_{L}^{(T, \tilde{a})}$. Si $k=n$, on a évidemment $c^{\prime}-\sum_{i=1}^{k} \frac{b_{i}^{\prime 2}}{4 a} \leqq 0$. 
Si $k<n$ et pour tout l'entier $i$ à $k+1 \leqq i \leqq n, b_{i}^{\prime}=0$, alors $c^{\prime}-\sum_{i=1}^{k} \frac{b_{i}^{\prime 2}}{4 a}$ $<0$. Si $k<n$ et $\left(b_{k+1}^{\prime}, \cdots, b_{n}^{\prime}\right) \neq(0, \cdots, 0)$, il existe un autre point $\tilde{a}^{\prime}$ $=\left(\tilde{a}_{1}^{\prime}, \cdots, \tilde{a}_{n}^{\prime}\right)$ de $\boldsymbol{R}^{n}$ tel que $\tilde{a}_{i}^{\prime}=-\frac{b_{i}^{\prime}}{2 a}(i=1, \cdots, k)$ et le terme constant de $L_{\widehat{a}^{\prime}}^{(T)}$ est $<0$. Par conséquent, si notre théorème est vrai dans le cas où

$$
L=a \sum_{i=1}^{\ell} \frac{\partial^{2}}{\partial x_{i}^{2}}+\sum_{i=\ell+1}^{n} b_{i} \frac{\partial}{\partial x_{i}}-c,
$$

où $a, b_{i}(i=\ell+1, \cdots, n)$ et $c$ sont de constantes vérifiant $a>0$ et $c \geqq 0$ ou bien $c>0$ d'accord avec $\ell=n$ ou bien $0 \leqq \ell<n$, alors, d'après la discussion inverse avec la présente réduction, on se comprend que, en général, notre théorème a lieu. Donc on suppose, dès maintenant, que $L$ est de la présente forme, et alors $G_{L}$ s'annule à l'infini. Pour tout $\tilde{p}=\left(p_{1}, \cdots, p_{m}\right)$ de $\bigcup_{m \geqq 1}\left(\boldsymbol{R}^{+}\right)^{m}, N_{\tilde{p}}$ s'annule aussi à l'infini, car $N_{\tilde{p}} * N_{\left(p_{1}, \cdots, p_{m}, 0\right)}$ $=G_{L}$, et par suite, pour tout $p>0, p \int d N_{\left(p_{1}, \cdots, p_{m}, p\right)} \leqq 1$. On connaît bien que $\tilde{C}_{R}\left(G_{L}\right)$ est vaguement fermé et que, pour tout l'élément $N_{p}$ de la résolvante associée au noyau $N$, il existe la classe divisible associée au noyau $N_{p}$ relativement au noyau $G_{L}$. Par conséquent, on peut supposer $\int d N<+\infty$. On désigne par $C_{0}=C_{0}\left(\boldsymbol{R}^{n}\right)$ l'espace de Banach constitué par toutes les fonctions finies et continues dans $\boldsymbol{R}^{n}$ s'annulant à l'infini et muni de la norme $\|\cdot\|$ de convergence uniforme. On note ensuite $\|\cdot\|^{\prime}$ la norme dans l'espace dual $C_{0}^{\prime}$ de $C_{0}$. Soit $\delta$ un nombre quelconque $>0$ donné. On peut montrer qu'il existe une suite croissante $\left(p_{m}\right)_{m=1}^{\infty}$ de nombres positifs vérifiant les deux conditions suivantes:

(a) Pour tout l'entier $m \geqq 1$, on a

$$
p_{1}>\frac{1}{\delta}, \quad \frac{p_{m}}{p_{m+1}}<\frac{1}{2}\left(\frac{\delta}{2^{m}}\right)^{1 / 2}
$$

(b) $d_{2}^{(1)}\left(p_{1}, p_{2}\right) / p_{1} p_{2}<\delta / 2$ et pour tous les entiers $k \geqq 0$ et $m \geqq 2$,

$$
\sum_{i=1}^{m}\left|\frac{d_{i}^{(m)}\left(p_{k+1}, \cdots, p_{k+m}\right)}{p_{k+i} \cdots p_{k+m}}-\frac{d_{i}^{(m+1)}\left(p_{k+1}, \cdots, p_{k+m+1}\right)}{p_{k+i} \cdots p_{k+m+1}}\right|<\delta / 2^{m}
$$

où $d_{m}^{(m)}\left(p_{k+1}, \cdots, p_{k+m}\right)=0$.

Cela est obtenu par récurrence, en utilisant le lemme 8. 
On note, pour deux entiers $k \geqq 0$ et $m \geqq 1, \widetilde{p}_{k, m}=\left(p_{k+1}, \cdots, p_{k+m}\right)$,

$$
\begin{aligned}
M_{\tilde{p}_{k}, m}^{(1)} & =p_{k+1} \cdots p_{k+m} N_{\left(0, p_{1}, \cdots, p_{k}\right)} * \cdots * N_{\left(0, p_{1}, \cdots, p_{k+m}\right)}, \\
M_{\tilde{p}_{k, m}}^{(2)} & =\sum_{i=1}^{m-1} d_{i}^{(m)}\left(p_{k+1}, \cdots, p_{k+m}\right) N_{\left(0, p_{1}, \cdots, p_{k+i}\right)} * \cdots * N_{\left(0, p_{1}, \cdots, p_{k+m}\right)}
\end{aligned}
$$

et

$$
M_{\tilde{p}_{k, m}}=M_{\tilde{p}_{k, m}}^{(1)}+M_{\tilde{p}_{k}, m}^{(2)}
$$

où $p_{0}=0$. On a alors, pour tout $m \geqq 2$,

$$
\begin{aligned}
& \| N_{\left(0, p_{1}, \cdots, p_{m-1}\right)} * N_{\left(0, p_{1}, \ldots, p_{m}\right)} *\left(\varepsilon-p_{m+1} N_{\left(0, p_{1}, \ldots, p_{m+1}\right)} \|^{\prime}\right. \\
& =\left\|N_{\left(0, p_{1}, \cdots, p_{m}\right)} * N_{\left(0, p_{1}, \ldots, p_{m+1}\right)} *\left(\varepsilon-p_{m} N_{\left(0, p_{1}, \cdots, p_{m}\right)}\right)\right\|^{\prime}<\frac{2}{p_{m} p_{m+1}},
\end{aligned}
$$

et donc

$$
\begin{aligned}
\left\|M_{\tilde{p}_{k}, m+1}-M_{\tilde{p}_{k}, m}\right\|^{\prime} \leqq & \left\|M_{\tilde{p}_{k}, m+1}^{(1)}-M_{\tilde{p}_{k, m}}^{(1)}\right\|^{\prime} \\
& +\sum_{i=1}^{m-1} \frac{d_{i}^{(m+1)}\left(p_{k+1}, \cdots, p_{k+m+1}\right)}{p_{k+i} \cdots p_{k+m+1}} \| p_{k+i} \cdots p_{k+m} N_{\left(0, p_{1}, \cdots, p_{k+i}\right)} \\
& * \cdots * N_{\left(0, p_{1}, \cdots, p_{k+m}\right)} *\left(\varepsilon-p_{k+m+1} N_{\left(0, p_{1}, \cdots, p_{k+m+1}\right)} \|^{\prime}\right. \\
+ & \sum_{i=1}^{m}\left|\frac{d_{i}^{(m+1)}\left(p_{k+1}, \cdots, p_{k+m+1}\right)}{p_{k+i} \cdots p_{k+m+1}}-\frac{d_{i}^{(m)}\left(p_{k+i}, \cdots, p_{k+m}\right)}{p_{k+i} \cdots p_{k+m}}\right| \\
& \times\left\|p_{k+i} \cdots p_{k+m} N_{\left(0, p_{1}, \cdots, p_{k+i}\right)} \cdots N_{\left(0, p_{1}, \cdots, p_{k+m}\right)}\right\|^{\prime} \\
& <\left(\frac{1}{2^{k}} \int d N_{\left(0, p_{1}, \cdots, p_{k}\right)}+\frac{\delta}{2^{k}}+1\right) \frac{\delta}{2^{m}}
\end{aligned}
$$

Donc, pour tout l'entier $k \geqq 0$, il existe une constante $a_{\tilde{\delta}, k} \geqq 0$ et un noyau de convolution $M_{\delta, k}$ sur $\boldsymbol{R}^{n}$ tels que

$$
\lim _{m \rightarrow+\infty} \sum_{i=1}^{m} \frac{d_{i}^{(m)}\left(p_{k+1}, \cdots, p_{k+m}\right)}{p_{k+i} \cdots p_{k+m}}=a_{\delta, k} \quad \text { et } \lim _{m \rightarrow+\infty}\left\|M_{\tilde{p}_{k, m}}-M_{\delta, k}\right\|^{\prime}=0 .
$$

On a encore $M_{\tilde{p}_{0}, 1}=p_{1} N * N_{\left(0, p_{1}\right)}$ et

$$
\begin{aligned}
\left\|M_{\tilde{p}_{0,1}}-M_{\delta, 0}\right\|^{\prime}= & \lim _{m \rightarrow \infty}\left\|M_{\tilde{p}_{0}, 1}-M_{\tilde{p}_{0}, m}\right\|^{\prime} \\
\leqq & \varlimsup_{m \rightarrow \infty}\left(\sum_{\ell=2}^{\infty}\left\|M_{\tilde{p}_{0}, \ell}^{(1)}-M_{\tilde{p}_{0}, \ell+1}^{(1)}\right\|^{\prime}+\left\|M_{\tilde{p}_{0}, 1}^{(1)}-M_{\tilde{p}_{0}, 2}^{(1)}\right\|^{\prime}\right. \\
& \left.+\sum_{i=1}^{m-1} \frac{d_{i}^{(m)}\left(p_{1}, \cdots, p_{m}\right)}{p_{i} \cdots p_{m}}\right) \\
\leqq & \left(\frac{1}{2} \int d N+2\right) \delta
\end{aligned}
$$


On obtient donc que $M_{\delta, 0}$ converge vaguement vers $N$ lorsque $\delta \downarrow 0$, et par suite il suffit de voir que $M_{\delta, 0}$ est de la forme

$$
M_{\delta, 0}=c_{\delta} \varepsilon+\int G_{L, p} d \nu_{\delta}(p),
$$

où $c_{\delta} \in \boldsymbol{R}^{+}$et $\nu_{\delta} \in M^{+}\left(\boldsymbol{R}^{+}\right)$. On a, pour tous les entiers $k \geqq 0$ et $m \geqq 3$,

$$
M_{\tilde{p}_{k, m}}=\left(p_{k+1}\right)^{2} \sum_{i=1}^{m-2} \frac{d_{i}^{(m-1)}\left(p_{k+2}, \cdots, p_{k+m}\right)}{p_{k+i+1} \cdots p_{k+m}} G_{L}+p_{k+1} G_{L} *\left(\varepsilon-p_{k+1} M_{\tilde{p}_{k+1}, m-1}\right),
$$

qui résulte de l'égalité

$$
\begin{aligned}
G_{L} *(\varepsilon- & \left.p_{k+i} \cdots p_{k+m} N_{\left(0, p_{1}, \cdots, p_{k+i}\right)} * \cdots * N_{\left(0, p_{1}, \cdots, p_{k+m}\right)}\right) \\
& =\sum_{s=0}^{m-i} \frac{p_{k+i} \cdots p_{k+m}}{p_{k+i} \cdots p_{k+i+s}} N_{\left(0, p_{1}, \cdots, p_{k+i+s-1}\right)} * \cdots * N_{\left(0, p_{1}, \cdots, p_{k+m}\right)}
\end{aligned}
$$

$\left(1 \leqq \forall i \leqq m\right.$ ) (cf. le lemme 7 ). $G_{L}$ s'annulant à l'infini, on a, pour tout l'entier $k \geqq 0$,

$$
M_{\delta, k}=\left(p_{k+1}\right)^{2} a_{\delta, k+1} G_{L}+p_{k+1} G_{L} *\left(\varepsilon-p_{k+1} M_{\delta, k+1}\right) .
$$

On obtient donc, pour tout l'entier $k \geqq 1$,

$$
0 \leqq L^{k} M_{\delta, 0}=p_{1}^{2} \cdots p_{k}^{2} M_{\delta, k}
$$

en dehors de l'origine. D'après la proposition 1 , pour tout l'entier $k \geqq 0$, la distribution

$$
(-1)^{k}\left(4 \pi^{2} a \sum_{i=1}^{\ell}\left|x_{i}\right|^{2}+2 \pi \sqrt{-1} \sum_{i=\ell+1}^{n} b_{i} x_{i}+c\right)^{k} \widehat{M_{\delta, 0}}(x) d x
$$

est de $C T P\left(R^{n}\right)$. On note, pour un entier $k \geqq 1, \tilde{M}_{\delta, 0}^{(k)}$ le noyau de convolution sur $\boldsymbol{R}^{n}$ définie par $\int f d \tilde{M}_{\delta, 0}^{(k)}=\int_{|x|>0} f(x) d\left(L^{k} M_{\delta, 0}\right)(x)$ pour toute $f$ de $C_{K}$. On peut supposer que $L$ n'est pas constant, et donc $G_{L}(\{0\})=0$, d'où $M_{\delta, k}(\{0\})=0 . \quad$ Par conséquent, $\tilde{M}_{\delta, 0}^{(k)}=p_{1}^{2} \cdots p_{k}^{2} M_{\delta, k} . \quad$ On obtient d'autre part,

$$
\begin{aligned}
& \left(4 \pi^{2} a \sum_{i=1}^{\ell}\left|x_{i}\right|^{2}+2 \pi \sqrt{-1} \sum_{i=\ell+1}^{n} b_{i} x_{i}+c\right) \widehat{M_{\delta, k}}(x) \\
& =\left(p_{k+1}\right)^{2} a_{\delta, k+1}+p_{k+1}\left(1-p_{k+1} \widehat{M_{\delta, k+1}}(x)\right) .
\end{aligned}
$$

De la même manière que dans le lemme 4 , il existe uniquement une constante $c_{\ell} \geqq 0$ et deux familles $\left(\alpha_{\sigma}^{(1)}\right)_{\sigma \in S_{1,0}^{(\ell)}},\left(\alpha_{\sigma}^{(2)}\right)_{\sigma \in S_{1,0}^{(\ell)}}$ de mesures positives 
sur $[\sqrt{c / a},+\infty)$ telle que, pour tout $\sigma$ de $S_{1,0}^{(\ell)}=\left\{\sigma=\left(\sigma_{1}, \cdots, \sigma_{n}\right) \in S_{1,0}\right.$; $\left.\sigma_{\ell+1}=\cdots=\sigma_{n}=0\right\}$,

$$
\widehat{M_{\delta, 0}}(r, \sigma)=c_{\ell}+\int \frac{1}{p+2 \pi \sqrt{-1} r} d \alpha_{\sigma}^{(1)}(p)+\int \frac{1}{p-2 \pi \sqrt{-1} r} d \alpha_{\sigma}^{(2)}(p)
$$

sur $\boldsymbol{R}^{+}$. De la même manière que dans le lemme 5 , on a, pour tout l'entier $k \geqq 1$ et pour tout $\sigma$ de $S_{1,0}^{(\ell)}$,

$$
\widehat{M_{\delta}^{(k)}(r)}(r)=\int \frac{\left(p^{2}-c / a\right)^{k}}{p+2 \pi \sqrt{-1} r} d \alpha_{\sigma}^{(1)}(p)+\int \frac{\left(p^{2}-c / a\right)^{k}}{p-2 \pi \sqrt{-1} r} d \alpha_{\sigma}^{(2)}(p)
$$

sur $\boldsymbol{R}^{+}$. Evidemment, pour tout l'entier $k \geqq 0, \int\left(p+\frac{1}{p}\right)^{k} d\left(\alpha_{\sigma}^{(1)}+\alpha_{\sigma}^{(2)}\right)(p)$ $<+\infty$. D'après le lemme 6 , on a, pour tout $\sigma$ de $S_{1,0}^{(\ell)}, \alpha_{\sigma}^{(1)}=\alpha_{\sigma}^{(2)}$, et donc, pour tout $\sigma$ de $S_{1,0}^{(\ell)}$, il existe une mesure positive $\nu_{\sigma}$ appartenant à $M^{+}\left(\boldsymbol{R}^{+}\right)$telle que

$$
\widehat{M_{\delta, 0}}(r, \sigma)=c_{\ell}+\int \frac{1}{p+4 \pi^{2} a r^{2}+c} d \nu_{\sigma}(p) \quad \text { sur } \boldsymbol{R}^{+}
$$

Posons, pour un point $\sigma$ de $S_{1,0}$,

$$
\varphi_{\sigma}(t)=\left\{\begin{array}{ll}
\widehat{M_{\delta, 0}}(t, \sigma) & \text { si } t \geqq 0 \\
\widehat{M_{\delta, 0}}(-t,-\sigma) & \text { si } t<0
\end{array} ;\right.
$$

alors, pour un point $\sigma=\left(\sigma_{1}, \cdots, \sigma_{n}\right)$ de $S_{1,0}$ vérifiant $\sigma_{1}=\cdots=\sigma_{\ell}=0$, la distribution $(-1)^{k}\left(2 \pi \sqrt{-1}\left(\sum_{i=k+1}^{n} b_{i} \sigma_{i}\right) t+c\right)^{k} \varphi_{\sigma}(t) d t$ est de $C T P\left(\boldsymbol{R}^{1}\right)$ pour tout l'entier $k \geqq 0$. La fonction $\varphi_{\sigma}$ étant finie, continue et de type positif, on obtient, d'après la proposition 1 et le lemme 2 , il existe une constante $c_{\sigma} \geqq 0$ et une mesure positive $\nu_{\sigma}$ sur $R^{+}$telles que

$$
\widehat{M_{\delta, 0}}(r, \sigma)=\varphi_{\sigma}(r)=c_{\sigma}+\int \frac{1}{p+2 \pi \sqrt{-1}\left(\sum_{i=\ell+1}^{n} b_{i} \sigma_{i}\right) r+c} d \nu_{\sigma}(p)
$$

sur $R^{+}$. Soient $\sigma=\left(\sigma_{1}, \cdots, \sigma_{n}\right)$ de $S_{1,0} ;$ posons $a_{\sigma}=a \sum_{i=1}^{\ell}\left|\sigma_{i}\right|^{2}$ et $b_{\sigma}=\sum_{i=\ell+1}^{n} b_{i} \sigma_{i}$ et supposons $a_{\sigma} \neq 0$. Si $b_{\sigma}=0$, alors, de la même manière que ci-dessus, on peut écrire 


$$
\widehat{M_{\tilde{\delta}, 0}}(r, \sigma)=c_{\ell}+\int \frac{1}{p+a_{\sigma} r^{2}+c} d \nu_{\sigma}(p),
$$

où $\nu_{\sigma} \in M^{+}\left(\dot{\boldsymbol{R}^{+}}\right)$. Supposons $b_{\sigma} \neq 0$; alors, d'après notre hypothèse concernant $L$, on a $c>0$. Pour tout l'entier $m \geqq 0$, on note

$$
\varphi_{\sigma}^{(m)}(t)=\left\{\begin{array}{ll}
\widehat{M_{\delta}^{(m)}}(t, \sigma) & \text { si } t \geqq 0 \\
\widehat{M_{\delta, 0}^{(m)}}(-t,-\sigma) & \text { si } t<0
\end{array},\right.
$$

où $\varphi_{\sigma}^{(0)}=\varphi_{\sigma}$. Soit $\kappa_{\sigma}^{(m)}$ le noyau de convolution sur $\boldsymbol{R}^{1}$ de masse totale finie tel que $\widehat{\kappa_{\sigma}^{(m)}}=\varphi_{\sigma}^{(m)}$. D'après le corollaire 1 dans [7], pour tous les entiers $m \geqq 0$ et $k \geqq 0$, la distribution $(-1)^{k}\left(4 \pi^{2} a_{\sigma} t^{2}+2 \pi \sqrt{-1} b_{\sigma} t+c\right)^{k} \varphi_{\sigma}^{(m)} d t$ est de $C T P\left(\boldsymbol{R}^{1}\right)$, et donc, d'après la proposition $1,\left(a_{\sigma} \frac{d^{2}}{d t^{2}}+b_{\sigma} \frac{d}{d t}-c\right)^{k} \kappa_{\sigma}^{(m)}$ est de $C P\left(\boldsymbol{R}^{1}\right)$. On a $\kappa_{\sigma}^{(m)}=\left(a_{\sigma} \frac{d^{2}}{d t^{2}}+b_{\sigma} \frac{d}{d t}-c\right) \kappa_{\sigma}^{(m-1)}$ en dehors de l'origine $(\forall m \geqq 1)$. D'après $c>0$, il existe un noyau de convolution de Hunt $G_{(\sigma)}$ sur $R^{1}$ de masse totale finie telle que $\left(a_{\sigma} \frac{d^{2}}{d t^{2}}+b_{\sigma} \frac{d}{d t}-c\right) G_{(\sigma)}=-\varepsilon$. Soient $\kappa_{\sigma}^{(m) \prime}$ et $G_{(\sigma)}^{\prime}$ les noyaux de convolution sur $\boldsymbol{R}^{1}$ définis par $d \kappa_{\sigma}^{(m) \prime}=\exp \left(\frac{b_{\sigma}}{2 a_{\sigma}} t\right) d \kappa_{\sigma}^{(m)}(t)$ et $d G_{(\sigma)}^{\prime}=\exp \left(\frac{b_{\sigma}}{2 a_{\sigma}} t\right) d G_{(\sigma)}(t)$, respectivement. Alors $G_{(\sigma)}^{\prime}$ est un noyau de convolution de Hunt sur $R^{1}$ et on a $\left(a_{o} \frac{d^{2}}{d t^{2}}\right.$ $\left.-\frac{b_{\sigma}^{2}}{4 a_{\sigma}}-c\right) G_{(\sigma)}^{\prime}=-\varepsilon . \quad$ Donc $\int d G_{(\sigma)}^{\prime}<+\infty . \quad$ Pour tout l'entier $m \geqq 0, \kappa_{\sigma}^{(m)}$ satisfaisant au principe de domination relatif au noyau $G_{(\sigma)}, \kappa_{\sigma}^{(m) \prime}$ satisfait aussi au principe de domination relatif au noyau $G_{(o)}^{\prime}$, et par suite $\int d \kappa_{\sigma}^{(m) \prime}<+\infty$. D'après le lemme 3 , pour tout l'entier $k \geqq 0,\left(a_{\sigma} \frac{d^{2}}{d t^{2}}\right.$ $\left.-\left(\frac{b_{\sigma}^{2}}{4 a_{\sigma}}+c\right)\right)^{k} \kappa_{\sigma}^{(m) \prime}$ est de $C P\left(\boldsymbol{R}^{1}\right)$. On a encore $\left(a_{\sigma} \frac{d^{2}}{d t^{2}}-\left(\frac{b_{\sigma}^{2}}{4 a_{\sigma}}+c\right)\right) \kappa_{\sigma}^{(m+1) \prime}$ $=\kappa_{\sigma}^{(m) \prime}$ en dehors de l'origine $\left({ }^{\forall} m \geqq 0\right)$. D'après les lemmes 4,5 , il existe uniquement une constante $c_{\sigma} \geqq 0$ et deux mesures positives $\alpha_{\sigma}^{(1)}, \alpha_{\sigma}^{(2)}$ sur $\left[\left(\frac{b^{2}}{4 a^{2}}+\frac{c}{a}\right)^{1 / 2},+\infty\right)$ telles que

$$
\widehat{\kappa_{\sigma}^{(0) \prime}}(t)=c_{\sigma}+\int \frac{1}{p+2 \pi \sqrt{-1} r} d \alpha_{\sigma}^{(1)}(p)+\int \frac{1}{p-2 \pi \sqrt{-1} t} d \alpha_{\sigma}^{(2)}(p)
$$


et que pour tout l'entier $m>0, \int\left(p+\frac{1}{p}\right)^{m} d\left(\alpha_{\sigma}^{(1)}+\alpha_{\sigma}^{(2)}\right)(p)<+\infty$,

$$
\widehat{\kappa_{\sigma}^{(m) \prime}}(t)=\int \frac{\left(p^{2}-d_{\sigma}\right)^{m}}{p+2 \pi \sqrt{-1} t} d \alpha_{\sigma}^{(1)}(p)+\int \frac{\left(p^{2}-d_{\sigma}\right)^{m}}{p-2 \pi \sqrt{-1} t} d \alpha_{\sigma}^{(2)}(p),
$$

où $d_{\sigma}=\frac{c}{a_{\sigma}}+\frac{b_{\sigma}^{2}}{4 a_{\sigma}^{2}}$. Ayant $M_{\delta, 0}^{(m)}=p_{1}^{2} \cdots p_{m}^{2} M_{\delta, m}$, on obtient

$$
\begin{aligned}
\left(4 \pi^{2} a_{\sigma} t^{2}\right. & \left.+2 \pi \sqrt{-1} b_{\sigma} t+c\right) \widehat{\kappa_{\sigma}^{(m)}}(t) \\
& =p_{1}^{2} \cdots p_{m}^{2}\left(\left(p_{m+1}\right)^{2} a_{\delta, m+1}+p_{m+1}\left(1-\frac{p_{m+1}}{p_{1}^{2} \cdots\left(p_{m+1}\right)^{2}} \widehat{\kappa_{\sigma}^{(m+1)}}(t)\right)\right)
\end{aligned}
$$

sur $\boldsymbol{R}^{1}$, et par suite

$$
\begin{aligned}
\left(4 \pi^{2} a_{\sigma} t^{2}\right. & \left.+a_{\sigma} d_{\sigma}\right) \widehat{\kappa_{\sigma}^{(m) \prime}}(t) \\
& =p_{1}^{2} \cdots\left(p_{m+1}\right)^{2}\left(a_{\delta, m+1}+\left(\frac{1}{p_{m+1}}-\frac{1}{p_{1}^{2} \cdots\left(p_{m+1}\right)^{2}}\right) \kappa_{\sigma}^{\widehat{(m+1) \prime}}(t)\right) .
\end{aligned}
$$

Par conséquent, pour tout l'entier $m \geqq 0$, la fonction $\left(4 \pi^{2} a_{\sigma} t^{2}+d_{\sigma}\right) \widehat{\kappa_{\sigma}^{(m) \prime}}(t)$ est bornée sur $R^{1}$. D'après le lemme 6 , on a $\alpha_{\sigma}^{(1)}=\alpha_{\sigma}^{(2)}$, et par suite il existe une mesure positive $\nu_{\sigma}$ sur $\boldsymbol{R}^{+}$telle que

$$
\widehat{\kappa_{\sigma}^{(0) \prime}}(t)=c_{\sigma}+\int \frac{1}{p+4 \pi^{2} a_{\sigma} t^{2}+c+b_{\sigma}^{2} / 4 a_{\sigma}} d \nu_{\sigma}(p)
$$

d'où

$$
\widehat{\kappa_{\sigma}^{(0)}}(t)=c_{\sigma}+\int \frac{1}{p+4 \pi^{2} a_{\sigma} t^{2}+2 \pi \sqrt{-1} b_{\sigma} t+c} d \nu_{\sigma}(p)
$$

On obtient ainsi qu'il existe une famille $\left(c_{\sigma}\right)_{\sigma \in S_{1,0}}$ de constantes $\geqq 0$ et une famille $\left(\nu_{o}\right)_{\sigma \in S_{1}, 0}$ dans $M^{+}\left(\boldsymbol{R}^{+}\right)$telles que

$$
\widehat{M_{\delta, 0}}(r, \sigma)=c_{\sigma}+\int \frac{1}{4 \pi^{2} a \sum_{i=1}^{\ell}\left|\sigma_{i}\right|^{2} r^{2}+2 \pi \sqrt{-1} \sum_{i=\ell+1}^{n} b_{i} \sigma_{i} r+c+p} d \nu_{\sigma}(p),
$$

où $\sigma=\left(\sigma_{1}, \cdots, \sigma_{n}\right)$. Ayant $M_{\delta, 0}(\{0\})=0$, on a, pour tout $\sigma$ de $S_{1,0}, c_{\sigma}=0$. On a

$$
\widehat{L M_{\delta, 0}}(r, \sigma)=\int\left(\frac{p}{4 \pi^{2} a \sum_{i=1}^{\ell}\left|\sigma_{i}\right|^{2} r^{2}+2 \pi \sqrt{-1} \sum_{i=\ell+1}^{n} b_{i} \sigma_{i} r+c+p}-1\right) d \nu_{\sigma}(p),
$$


et d'après le lemme 7 et l'unicité de la décomposition dans le théorème de Levy-Khinchine, on a $\int d \nu_{\sigma}<+\infty\left({ }^{\forall} \in S_{1,0}\right)$ et

$$
\widehat{M_{\delta}^{(1)}(r, \sigma)}=\int \frac{p}{4 \pi^{2} a \sum_{i=1}^{\ell}\left|\sigma_{i}\right|^{2} r^{2}+2 \pi \sqrt{-1} \sum_{i=\ell+1}^{n} b_{i} \sigma_{i} r+c+p} d \nu_{\sigma}(p)
$$

Par récurrence, on a, pour tout l'entier $m \geqq 1$,

$$
\widehat{M_{\delta, 0}^{(m)}}(r, \sigma)=\int \frac{p^{m}}{4 \pi^{2} a \sum_{i=1}^{\ell}\left|\sigma_{i}\right|^{2} r^{2}+2 \pi \sqrt{-1} \sum_{i=\ell+1}^{n} b_{i} \sigma_{i} r+c+p} d \nu_{\sigma}(p) .
$$

D'après $\int d M_{\delta, 0}^{(m)}<+\infty, \widehat{M}_{\delta, 0}^{(m)}$ est finie et continue, et par suite $\int \frac{p^{m}}{c+p} d \nu_{\sigma}(p)$ est finie et ne depend pas de $\sigma$. De la même manière que dans le lmeme $6, \nu_{\sigma}$ ne depend pas de $\sigma$. Posons $\nu=\nu_{\sigma}$; alors on obtient

$$
\widehat{M}_{\delta, 0}(x)=\int \frac{1}{4 \pi^{2} a \sum_{i=1}^{\ell}\left|x_{i}\right|^{2}+2 \pi \sqrt{-1} \sum_{i=\ell+1}^{n} b_{i} x_{i}+c+p} d \nu(p)
$$

sur $\boldsymbol{R}^{n}$, où $x=\left(x_{1}, \cdots, x_{n}\right)$. Par conséquent on a $M_{\delta, 0}=\int G_{L, p} d \nu(x)$.

La démonstration est ainsi complète.

On désigne par $C_{R}\left(G_{L}\right)$ le cône convexe divisible relatif au noyau $G_{L}$. De la même manière que dans [6], l'ensemble des points extrêmes dans $C_{R}\left(G_{L}\right)$ est égal à $\{\varepsilon\} \cup\{0\} \cup\left\{G_{L, p} ; p \geqq 0\right\}$. D'après le théorème 1 , si $L$ est uniformément elliptique, alors tout l'élément de $C_{R}\left(G_{L}\right)$ est analytique en dehors de l'origine.

\section{BIBLIOGRAPHIE}

[1] G. Choquet et J. Deny: Aspects linéaires de la théorie du potentiel, Noyaux de composition satisfaisant au principe du balayage sur tout ouvert, C. R. Acad. Sci. Paris, 250 (1960), p. 4260-4262.

[2] J. Deny: Noyaux de convolution de Hunt et noyaux associés à une famille fondamentale, Ann. Inst. Fourier, 12 (1962), p. 643-667.

[ 3 ] C. S. Herz: Analyse harmonique à plusieurs variables, Sém. Math. d'Orsay, $1965 / 66$.

[4] M. Itô: Sur le principe de domination pour les noyaux de convolution, Nagoya Math. J., 50 (1973), p. 149-173.

[5] - Une caracterisation du principe de domination pour les noyaux de convolution, Japan. J. Math., 1 (1975), p. 5-35. 
[6] - - Sur les cônes convexes de Riesz et les noyaux de convolution complètement sous-harmoniques, Nagoya Math. J., 55 (1974), p. 111-144.

[7] - : Sur L'unicuté du cône convexe divisible constitué par de noyaux de convolution de Dirichlet, Nagoya Math. J., 57 (1975), p. 127-152.

[ 8 ] D. Widder: The Laplace Transform, Princeton Univ. Press, Princeton, 1948.

Université de Nagoya 